\title{
The Synergy Between Qualitative Theory, Quantitative Calculations, and Direct Experiments in Understanding, Calculating, and Measuring the Energy Differences Between the Lowest Singlet and Triplet States of Organic Diradicals
}

\author{
W. Carl Lineberger*a and Weston Thatcher Borden*b
}

JILA and the Department of Chemistry and Biochemistry, University of Colorado, and the National Institute of Standards and Technology, Boulder, CO 80309-0440, and the Department of Chemistry and Center for Advanced Simulation, Computation and Modeling, University of North Texas, Denton, TX 76203-5070, USA

\begin{abstract}
This Perspective describes research, carried out in the authors' labs over the past forty years, aimed at understanding, predicting, and measuring the singlet-triplet energy differences $\left(\Delta E_{\mathrm{ST}}\right)$ in diradicals. A theory for qualitatively predicting the ground states of diradicals and the use of Negative Ion Photoelectron Spectroscopy (NIPES) for measuring $\Delta E_{\mathrm{ST}}$ are described. The application of this theory, $a b$ initio calculations, and NIPES to the prediction and measurement of $\Delta E_{\mathrm{ST}}$ in a wide variety of organic diradicals is detailed. Among the diradicals that are discussed in this Perspective are $\mathrm{HN}, \mathrm{CH}_{3} \mathrm{~N}, \mathrm{PhN}, \mathrm{CH}_{2}$, trimethylenemethane (TMM), oxyallyl (OXA), meta-benzoquinodimethane (MBQDM), meta-benzoquinone (MBQ),

tetramethyleneethane (TME), 1,2,4,5-tetramethylenebenzene (TMB), and $D_{8 \mathrm{~h}}$ cyclooctateraene (COT). All of these diradicals have been studied in one and, in most cases, in both of the authors' laboratories. The studies of OXA and $D_{8 \mathrm{~h}}$ COT were, in fact, collaborations between the research groups of the authors. These two projects both took advantage of the ability of NIPES to provide information about transition states. Transition-state spectroscopy was used to measure the cabonyl stretching frequency in the singlet state of OXA and to establish that $D_{8 \mathrm{~h}}$ COT violates the strictest version of Hund's rule.
\end{abstract}

\footnotetext{
${ }^{a}$ University of Colorado. ${ }^{b}$ University of North Texas
} 


\section{Introduction}

Successful qualitative theories have two important functions. First, theories provide interpretations of the results of experiments and/or calculations that have already been performed. Second, the predictions made by the theories motivate both experimentalists and computational chemists to attempt to validate the theories by testing the predictions made by them.

However, in some cases, it is not qualitative theories but the results of high-level calculations that motivate experimentalists to test how quantitatively accurate the calculations are. In other cases calculations are performed to see how well they reproduce experimental results that have already been obtained. When the results of calculations and experiments are found to be at odds with each other, additional experiments and/or calculations almost inevitably follow.

Investigations of the energy differences between the lowest singlet and triplet state $\left(\Delta E_{\mathrm{ST}}\right)$ of organic diradicals ${ }^{1}$ have provided many illustrations of the above types of synergies. Qualitative theories have resulted in computational and/or experimental tests, and calculations and experiments have not only stimulated each other but, in some cases, also led to the development of qualitative theories.

For example, in response to the results of experiments and calculations on cyclobutadiene and trimethylenemethane, one of the coauthors of this review (WTB) helped to develop a qualitative theory, which allows the qualitative prediction of the approximate size and sign of $\Delta E_{\mathrm{ST}}$ in organic diradicals. ${ }^{1,2}$ Subsequently, he and his collaborators carried out ab initio calculations on a wide variety of diradicals, in order to test the qualitative theory and to predict quantitatively the values of $\Delta E_{\mathrm{ST}}$ in the diradicals. The qualitative theory and the results of these $a b$ initio calculations are described in this Perspective.

The other coauthor of this review (WCL) developed a technique called Negative Ion Photoelectron Spectroscopy (NIPES), which allows the very accurate measurement $\Delta E_{\mathrm{ST}}$ in diradicals. ${ }^{3}$ He and his collaborators have used NIPES to measure the singlet-triplet energy differences in many of the diradicals, for which predictions of $\Delta E_{\mathrm{ST}}$ had already been made., 5

In some cases the values of $\Delta E_{\mathrm{ST}}$ that were obtained by these experiments served to stimulate more calculations and, at least in the case of methylene $\left(\mathrm{CH}_{2}\right)$, more experiments. The results of these NIPES experiments are also described in this perspective.

The coauthors of this review have also collaborated with each other in designing and interpreting experiments that led to the measurement of $\Delta E_{\mathrm{ST}}$ in two organic diradicals, oxyallyl $(\mathrm{OXA})^{6}$ and $D_{8 \mathrm{~h}}$ cyclooctatetraene (COT). ${ }^{7}$ The NIPES experiments were carried out in WCL's $\mathrm{lab}$, and the interpretations of the results of these experiments were aided by calculations that were performed by WTB's research group.

Each of these collaborative NIPES experiments provided spectroscopic information about what turned out to be a transition state. ${ }^{8}$ Transition-state spectroscopy confirmed the prediction of a high $\mathrm{C}=\mathrm{O}$ stretching frequency in singlet OXA, a species that was shown by both the NIPE spectrum and by $a b$ initio calculations to be the transition state for the ring opening of cyclopropanone. ${ }^{6}$ Transition-state spectroscopy also confirmed the prediction, made by both qualitative theory and quantitative calculations, of a violation of the strictest form of Hund's rule $^{9-12}$ in $D_{8 \mathrm{~h}}$ cyclooctatetraene ${ }^{7}$. 
This Perspective describes the authors' individual and collaborative research on singlettriplet energy differences in diradicals. The Perspective has been divided into five sections. Following this introductory section, Section 2 provides a review of the wave functions for the singlet and triplet states of diradicals ${ }^{1}$ and of the topological theory for estimating the size and sign of $\Delta E_{\mathrm{ST}}$ between these spin states. ${ }^{2}$ Section 3 describes NIPES of radical anions and how this technique allows $\Delta E_{\mathrm{ST}}$ in neutral diradicals to be measured. ${ }^{3}$

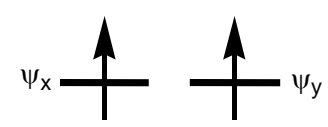

(a)

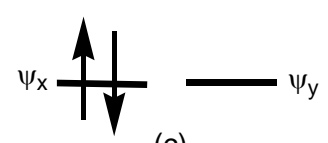

(c)

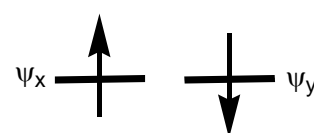

(e)

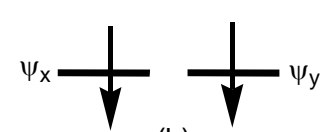

(b)

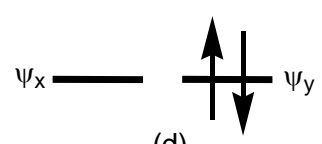

(d)

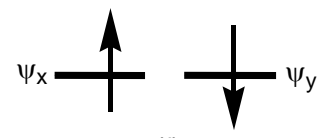

(f)

Figure 1 The six possible electronic configurations that arise from distributing two electrons among two orbitals.

Those readers who are already familiar with the material in these two sections may wish to skip one or both of them and to proceed directly to Section 4 . This section, which is the longest in this Perspective, provides descriptions of the calculation and measurement of $\Delta E_{\mathrm{ST}}$ in a variety of diradicals, including nitrenes $\left(\mathrm{HN}, \mathrm{CH}_{3} \mathrm{~N}\right.$, and $\left.\mathrm{PhN}\right)$, methylene $\left(\mathrm{CH}_{2}\right)$, trimethylenemethane (TMM), oxyallyl (OXA), $m$-benzoquinodimethane (MBQDM), $m$ benzoquinone (MBQ), tetramethyleneethane (TME), 1,2,4,5-tetramethylenebenzene (TMB), and $D_{8 \mathrm{~h}}$ cyclooctatetraene (COT). Section 5 provides a brief summary of the results described in Section 4 and highlights again the synergies between theory, calculations, and experiments in establishing the values of $\Delta E_{\mathrm{ST}}$ in the diradicals discussed in this Perspective.

\section{Wave Functions for Diradicals and the Qualitative Prediction of the Relative Energies of the Lowest Singlet and Triplet States in Them.}

An atom or molecule in which two electrons occupy two orbitals of the same or nearly the same has been defined as a diradical. ${ }^{11}{ }^{13}$ Some chemists prefer the name "biradical", ${ }^{14}$ but others call molecules with two positive charges "dications" and molecules with two negative charges "dianions". Therefore, calling molecules that, at least potentially, have two unpaired electrons "diradicals" has the virtue of a consistency.

2.1. Electronic Configurations in Diradicals. As shown in Figure 1, there are six possible ways of distributing two electrons between two orbitals, $\psi_{\mathrm{x}}$ and $\psi_{\mathrm{y}}$. Configurations (a) and (b) are the $S_{z}= \pm 1$ components of a triplet; whereas, configurations (c) and (d) are obviously both singlets. Like configurations (c) and (d), configurations (e) and (f) each have $S_{\mathrm{z}}=0$, but the latter pair of configurations each have a spin quantum number of $\left\langle S^{2}\right\rangle=1$. Since triplet states 
have $S^{2}=2$, and singlet states have $S^{2}=0$, configurations (e) and (f) are not pure spin states. Instead, each of these configurations is a 1:1 combination of a singlet and a triplet state.

It is easy to demonstrate that the linear combination of configurations (e) and (f) in eq. 1,

$$
\begin{aligned}
\Psi_{\text {TO }} & =\frac{\left(\left|\psi_{x}^{\alpha}(1) \psi_{y}^{\beta}(2)\right\rangle+\left|\psi_{x}^{\beta}(1) \psi_{y}^{\alpha}(2)\right\rangle\right)}{\sqrt{2}} \\
& =\frac{\left[\psi_{x}(1) \psi_{y}(2)-\psi_{y}(1) \psi_{x}(2)\right][\alpha(1) \beta(2)+\beta(1) \alpha(2)]}{2}
\end{aligned}
$$

is a triplet state, $\Psi_{\mathrm{T} 0}$, with $S_{\mathrm{z}}=0$. [The Dirac "kets" (|..>) in the first line of eq. (1) indicate properly antisymmetrized two-electron wave functions]. Operating on $\Psi_{\mathrm{T} 0}$ with the $S^{2}$ operator shows that, like the wavefunctions $\Psi_{\mathrm{T}+1}$ for configuration (a) and $\Psi_{\mathrm{T}-1}$ for configuration (b), $\Psi_{\mathrm{T} 0}$ has an eigenvalue of $S^{2}=2$.

The spatial wavefunction for all three components of the triplet state -- $\Psi_{\mathrm{T}+1}, \Psi_{\mathrm{T} 0}$, and $\Psi_{\mathrm{T}-1}$, changes sign on permutation of the labels, (1) and (2), on the two electrons, but the spin wave functions -- $\alpha(1) \alpha(2), \beta(1) \beta(2)$, and $[\alpha(1) \beta(2)+\beta(1) \alpha(2)] / \sqrt{ } 2$ do not. Thus, each of the components of the triplet state has an antisymmetric spatial wavefunction but a symmetric spin wavefunction.

The other linear combination of configurations (e) and (f)

$$
\begin{aligned}
\Psi_{\text {so }} & =\frac{\left(\left|\psi_{x}^{\alpha}(1) \psi_{y}^{\beta}(2)\right\rangle-\left|\psi_{x}^{\beta}(1) \psi_{y}^{\alpha}(2)\right\rangle\right)}{\sqrt{2}} \\
& =\frac{\left[\psi_{x}(1) \psi_{y}(2)+\psi_{y}(1) \psi_{x}(2)\right][\alpha(1) \beta(2)-\beta(1) \alpha(2)]}{2}
\end{aligned}
$$

has $S^{2}=0$; so it is a singlet. In contrast to the three components of $\Psi_{\mathrm{T}}, \Psi_{\mathrm{S} 0}$ has a symmetric spatial wavefunction, which does not changes sign on permutation of the labels on the two electrons, and an antisymmetric spin wavefunction, $[\alpha(1) \beta(2)-\beta(1) \alpha(2)] / \sqrt{ } 2$, which does.

2.2. Relative Energies of the Triplet and the "Open-Shell" Singlet State. In the absence of a magnetic field, and ignoring the small dipolar coupling between the unpaired electrons, all three components of the triplet, $\Psi_{\mathrm{T}+1}, \Psi_{\mathrm{T} 0}$, and $\Psi_{\mathrm{T}-1}$ have the same energy,

$$
E_{T}=\varepsilon\left(\psi_{x}\right)+\varepsilon\left(\psi_{y}\right)+J_{x y}-K_{x y}
$$

where $\varepsilon\left(\psi_{\mathrm{x}}\right)$ and $\varepsilon\left(\psi_{\mathrm{y}}\right)$ are, respectively, the energy of one electron in $\psi_{\mathrm{x}}$ and one in $\psi_{\mathrm{y}}$, exclusive of the Coulombic interaction of the electrons with each other. The Coulombic interaction between the charges due to an electron in $\psi_{\mathrm{x}}$ and one in $\psi_{\mathrm{y}}$ is given by

$$
J_{x y}=\iint \psi_{x}^{2}(1) \frac{e^{2}}{r_{12}} \psi_{y}^{2}(2)
$$

In the triplet state, the Coulombic interaction energy between the charge distributions, given by $\psi_{\mathrm{x}}{ }^{2}(1) \mathrm{e}$ and by $\psi_{\mathrm{y}}{ }^{2}(2) \mathrm{e}$, must be corrected for the antisymmetrization of the spatial wave function for the triplet, $\psi_{\mathrm{x}}(1) \psi_{\mathrm{y}}(2)-\psi_{\mathrm{y}}(1) \psi_{\mathrm{x}}(2)$. It is easy to see that this spatial wave function vanishes if (a) both electrons occupy the same $\mathrm{MO}$ or (b) if the electrons in $\psi_{\mathrm{x}}$ and $\psi_{\mathrm{y}}$ 
are at the same point in space. Situation (a) gives rise to the familiar statement of the Pauli exclusion principle - two electrons of the same spin cannot occupy the same orbital. Situation (b) gives rise to the less familiar, but no less important, statement that the probability of two electrons of the same spin simultaneously being at the same point in space is zero.

Because the anti-symmetric spatial wave for two electrons of the same spin prevents them from simultaneously appear the same region of space, in the triplet the Coulombic interaction between the charge distributions, given by $\psi_{\mathrm{x}}{ }^{2}(1) \mathrm{e}$ and $\psi_{\mathrm{y}}{ }^{2}(2) \mathrm{e}$, must be corrected. This correction results in the so-called exchange integral, $\mathrm{K}_{\mathrm{xy}}$, appearing in eq. 3 with a minus sign. where

$$
K_{x y}=\iint \psi_{x}(1) \psi_{y}(1) \frac{e^{2}}{r_{12}} \psi_{x}(2) \psi_{y}(2)
$$

It should be noted that, if the electrons in $\psi_{\mathrm{x}}$ and $\psi_{\mathrm{y}}$ have the same coordinates, $\mathrm{J}_{\mathrm{xy}}-\mathrm{K}_{\mathrm{xy}}$ vanishes. Therefore, in eq. 3 terms in $\mathrm{J}_{\mathrm{xy}}$ that correspond to the Coulombic repulsion energy when the two electrons are very close to each other, are cancelled by terms of similar size in $-\mathrm{K}_{\mathrm{xy}}$.

Unlike the case in the triplet wave function in eq. 1, in the singlet wave function, $\Psi_{\mathrm{S} 0}$, in eq. 2 there is no prohibition against the electrons in $\psi_{\mathrm{x}}$ and $\psi_{\mathrm{y}}$ having the same coordinates. In fact, on squaring the spatial part, $\left[\psi_{\mathrm{x}}(1) \psi_{\mathrm{y}}(2)+\psi_{\mathrm{y}}(1) \psi_{\mathrm{x}}(2)\right] / \sqrt{2}$, of the "open-shell" singlet wave function, $\Psi_{\mathrm{S} 0}$, in eq. 2 the cross term doubles the probability that two electrons will have the same set of coordinates, compared to the probability given by the product of $\psi_{\mathrm{x}}{ }^{2}(1)$ and $\psi_{\mathrm{y}}{ }^{2}(2)$. Therefore, if the electrons of the same spin in $\Psi_{\mathrm{T}}$ can be said to have their motions "correlated", so that they tend to avoid each other, the electrons of the same spin in $\Psi_{\mathrm{S} 0}$ can be said to have their motions "anticorrelated", so that they tend to be found together in the same region of space. The appearance with a positive sign of $\mathrm{K}_{\mathrm{xy}}$ in the expression for the energy of $\Psi_{\mathrm{S} 0}$,

$$
E_{S O}=\varepsilon\left(\psi_{x}\right)+\varepsilon\left(\psi_{x}\right)+J_{x y}+K_{x y}
$$

accounts for the effect of this anti-correlation on the Coulombic repulsion energy in the "openshell" singlet state, in which one electron occupies $\psi_{\mathrm{x}}$ and another electron of opposite spin occupies $\psi_{\mathrm{y}}$.

Taken together, eqs. 3 and 6 predict that, if the optimal MOs, $\psi_{\mathrm{x}}$ and $\psi_{\mathrm{y}}$, for the triplet and the open-shell singlet are the same, the energy of the triplet will be $2 \mathrm{~K}_{\mathrm{xy}}$ lower than that of the open-shell singlet. However, the term $+K_{x y}$ in eq. 6 penalizes $\Psi_{S}$ for having regions of space where $\psi_{\mathrm{x}}$ and $\psi_{\mathrm{y}}$ overlap. Therefore, the optimal MOs, $\psi_{\mathrm{x}}{ }^{\mathrm{S}}$ and $\psi_{\mathrm{y}}{ }^{\mathrm{S}}$, for the open-shell singlet may not necessarily be the same as the optimal MOs, $\psi_{\mathrm{x}}{ }^{\mathrm{T}}$ and $\psi_{\mathrm{y}}{ }^{\mathrm{T}}$, for the triplet. Unlike the MOs for the triplet state, the optimal MOs for the open-shell singlet will tend to be localized in different regions of space, so that $\psi_{\mathrm{x}}{ }^{\mathrm{S}}$ and $\psi_{\mathrm{y}}{ }^{\mathrm{s}}$ do not overlap and, consequently, so that $\mathrm{K}_{\mathrm{xy}}{ }^{\mathrm{s}}$ is small.

Stated in physical terms, the electrons in the open-shell singlet cannot avoid each other in those regions of space where the MOs, $\psi_{\mathrm{x}}{ }^{\mathrm{S}}$ and $\psi_{\mathrm{y}} \mathrm{s}$, overlap. Consequently, the only way that the electrons in $\Psi_{\mathrm{S} 0}$ can reduce their mutual Coulombic repulsion is to become localized in different regions of space, in order to minimize the regions of space that the MOs, $\psi_{\mathrm{x}}{ }^{\mathrm{S}}$ and $\psi_{\mathrm{y}}{ }^{\mathrm{S}}$, have in common.

It should be noted that, even if localization of the electrons of opposite spin to different regions of space in $\psi_{\mathrm{x}} \mathrm{S}$ and $\psi_{\mathrm{y}}{ }^{\mathrm{S}}$ makes the Coulombic repulsion energy in the singlet 
approximately the same as that in the triplet, any modification of the optimal triplet MOs, $\psi_{\mathrm{x}}{ }^{\mathrm{T}}$ and $\psi_{\mathrm{y}}{ }^{\mathrm{T}}$, that is required in order to achieve $\mathrm{K}_{\mathrm{xy}} \mathrm{S} \approx 0$ for $\psi_{\mathrm{x}}{ }^{\mathrm{S}}$ and $\psi_{\mathrm{y}} \mathrm{S}$, will tend to make the energy of the singlet state higher than that of the triplet. Therefore, the only circumstances under which an "open-shell" singlet state can have approximately the same energy as the corresponding triplet is if the optimal MOs for the triplet are confined to different regions of space; i.e., if $\psi_{\mathrm{x}}{ }^{\mathrm{T}}$ and $\psi_{\mathrm{y}}{ }^{\mathrm{T}}$ are disjoint. $^{2}$

If the optimal MOs for the triplet state are disjoint, then $K_{\mathrm{xy}} \approx 0$ in eq. 5 ; so $E_{\mathrm{S} 0}$ in eq. 6 is approximately the same as $E_{\mathrm{T}}$ in eq. 3. Coulombic interactions of the electrons in $\psi_{\mathrm{x}}$ and $\psi_{\mathrm{y}}$ with the electrons in doubly-occupied MOs generally are smaller for the "open-shell" singlet than for the triplet (vide infra). Therefore, if the optimal MOs for the triplet are disjoint, the energy of the "open-shell" singlet state can actually be lower than that of the triplet; and a violation of Hund's rule is possible. ${ }^{9-12}$ While higher level calculations certainly improve upon the description presented here, the essential physical processes are well explained at this level.

2.3. Relative Energies of "Open-Shell" and "Closed-Shell" Singlet States. $\Psi_{\mathrm{S}}$, , the open-shell singlet wave function, is only one of the three singlet wave functions that can be formed by distributing two electrons among two orbitals. If $\psi_{\mathrm{x}}$ and $\psi_{\mathrm{y}}$ have exactly the same energy, then the other two singlet wave functions are the linear combinations of the two "closed shell" configurations (c) and (d) in eq. 7.

$$
\Psi_{T C}=\frac{\left(\left|\psi_{x}^{2}\right\rangle \pm\left|\psi_{y}^{2}\right\rangle\right)}{\sqrt{2}}
$$

The energies of this pair of two-configuration (TC) singlet wave functions are given by

$$
E_{ \pm}=\varepsilon\left(\psi_{x}\right)+\varepsilon\left(\psi_{y}\right)+\frac{J_{x x}+J_{y y}}{2} \pm K_{x y}
$$

where $\mathrm{E}_{-}$is the energy of the TC singlet wave function with the minus sign in eq. 7 . According to eq. 8, the energy of this TC wave function is lower than that of the TC wave function with a plus sign by $2 \mathrm{~K}_{\mathrm{xy}}$. Henceforth, $\Psi_{\mathrm{TC}}$ will be used exclusively to refer to the lower energy of the twoconfiguration wave functions in eq. 7.

Assuming that the singlet wave functions, $\Psi_{\mathrm{S}}$ and $\Psi_{\mathrm{TC}}$ in eqs 2 and 7 have the same set of optimal MOs, $\psi_{\mathrm{x}}$ and $\psi_{\mathrm{y}}$, and comparing the energy of the "open-shell" singlet in eq. (6) with that of the "closed-shell", TC singlet in eq. (8), the energy difference is

$$
E_{S O}-E_{T C}=J_{x y}-\frac{\left(J_{x x}+J_{y y}\right)}{2}+2 K_{x y}
$$

In some diradicals symmetry requires that $\Psi_{\mathrm{S} 0}$ and $\Psi_{\mathrm{TC}}$ are degenerate in energy, which means that $\left(\mathrm{J}_{\mathrm{xx}}+\mathrm{J}_{\mathrm{yy}}\right) / 2-\mathrm{J}_{\mathrm{xy}}=2 \mathrm{~K}_{\mathrm{xy}}$. However, if symmetry does not make $\Psi_{\mathrm{s} 0}$ and $\Psi_{\mathrm{TC}}$ degenerate, then the question arises as to which wave function is lower in energy. 
The answer depends on the choice of a set of degenerate MOs. If $\psi_{\mathrm{x}}$ and $\psi_{\mathrm{y}}$ are degenerate in energy, then any pair of orthogonal linear combinations of these two MOs is also an acceptable choice for the degenerate MOs. Thus, there are an infinite number of possible choices for the degenerate MOs in a diradical.

For example, the lower-energy, TC, singlet wave function in eq. 7, can be factored as shown in eq. 10

$$
\begin{aligned}
\Psi_{T C} & =\frac{\left(\left|\psi_{x}^{2}\right\rangle-\left|\psi_{y}^{2}\right\rangle\right)}{\sqrt{2}} \\
& =\frac{\left|\left(\psi_{x}+\psi_{y}\right)^{\alpha}(1)\right\rangle\left|\left(\psi_{x}-\psi_{y}\right)^{\beta}(2)\right\rangle-\left|\left(\psi_{x}+\psi_{y}\right)^{\beta}(1)\right\rangle\left|\left(\psi_{x}-\psi_{y}\right)^{\alpha}(2)\right\rangle}{2 \sqrt{2}} \\
& =\frac{\left[\left(\psi_{x}+\psi_{y}\right)(1)\left(\psi_{x}-\psi_{y}\right)(2)+\left(\psi_{x}+\psi_{y}\right)(2)\left(\psi_{x}-\psi_{y}\right)(1)\right][\alpha(1) \beta(2)-\beta(1) \alpha(2)]}{4}
\end{aligned}
$$

If a new set of degenerate orbitals is defined as

$$
\psi_{x^{\prime}}=\frac{\psi_{x}+\psi_{y}}{\sqrt{2}}
$$

and

$$
\psi_{y^{\prime}}=\frac{\psi_{x}-\psi_{y}}{\sqrt{2}}
$$

then $\Psi_{\mathrm{TC}}$ in eq. 10 can be written in terms of the new set of orbitals $\psi_{\mathrm{x}^{\prime}}$ and $\psi_{\mathrm{y}^{\prime}}$ as

$$
\begin{aligned}
\Psi_{T C} & =\frac{\left|\psi_{x^{\prime}}^{\alpha}(1) \psi_{y^{\prime}}^{\beta}(2)\right\rangle-\left|\psi_{x^{\prime}}^{\beta}(1) \psi_{y^{\prime}}^{\alpha}(2)\right\rangle}{\sqrt{2}} \\
& =\frac{\left[\psi_{x^{\prime}}(1) \psi_{y^{\prime}}(2)+\psi_{x^{\prime}}(2) \psi_{y^{\prime}}(1)\right][\alpha(1) \beta(2)-\beta(1) \alpha(2)]}{2}=\Psi_{S O^{\prime}}
\end{aligned}
$$

By defining a new set of degenerate MOs in eqs. (11) and (12), the TC, "closed-shell", singlet wave function, $\Psi_{\mathrm{TC}}$, in eq. 10 , has been transformed into an "open-shell" singlet wave function, $\Psi_{\mathrm{S} 0}{ }^{\prime}$, in eq. (13). Clearly, if one wishes to use the terms "open-shell" and "closed-shell" to describe the two, lowest-energy. singlet wave functions for a diradical, one must specify the set of degenerate MOs upon which these names are based.

2.4. GVB Orbitals. The primed pair of degenerate MOs in eqs. 11 and 12 are often referred to as the "generalized valence-bond" (GVB) orbitals. ${ }^{15}$ By using the GVB orbitals, as defined in eqs. 11 and 12, the TC wave function in eq. 10, can be rewritten as an "open-shell" singlet wave function. Doing so is conceptually advantageous for two reasons. First, an openshell singlet wave function, like $\Psi_{\mathrm{S} 0^{\prime}}$, in eq. 13 , has a much more transparent physical interpretation than a two-configuration wave function, like $\Psi_{\mathrm{TC}}$, in eq 10 . Second, the transformation to GVB orbitals provides an easy way of qualitatively comparing the energy of the "closed-shell", singlet wave function, $\Psi_{\mathrm{TC}}$, in eq. 10 with that of the "open-shell" singlet wave function, $\Psi_{\mathrm{S} 0}$, in eq. 2 , in order to determine which singlet state is lower in energy. 
2.4.1. GVB MOs of $\mathbf{N H}$. In order to clarify the relationship between a pair of nonbonding (NB)MOs for a diradical and the GVB orbitals derived from them, it is useful to discuss some specific examples. We begin with the HN diradical.

The degenerate NBMOs for this diradical can be chosen to be the $2 p_{x}$ and $2 p_{y}$ AOs on $N$ that are shown in Fig. 2a. The "open-shell" singlet wave function for $\mathrm{HN}$ is then

$$
\begin{aligned}
\Psi_{\text {SO }} & =\frac{\left(\left|\ldots 2 p_{x}^{\alpha}(1) 2 p_{y}^{\beta}(2)\right\rangle-\left|\ldots 2 p_{x}^{\beta}(1) 2 p_{y}^{\alpha}(2)\right\rangle\right)}{\sqrt{2}} \\
& =\frac{\left[\ldots 2 p_{x}(1) 2 p_{y}(2)+\ldots 2 p_{y}(1) 2 p_{x}(2)\right][\alpha(1) \beta(2)-\beta(1) \alpha(2)]}{2}
\end{aligned}
$$

Transforming the two-configuration, "closed-shell" singlet wave function for HN to GVB orbitals gives

$$
\begin{aligned}
\Psi_{T C} & =\frac{\left(\left|\ldots 2 p_{x}^{2}\right\rangle-\left|\ldots 2 p_{y}^{2}\right\rangle\right)}{\sqrt{2}} \\
& =\frac{\left|\ldots 2 p_{x^{\prime}}^{\alpha}(1) 2 p_{y^{\prime}}^{\beta}(2)\right\rangle-\left|\ldots 2 p_{x^{\prime}}^{\beta}(1) 2 p_{y^{\prime}}^{\alpha}(2)\right\rangle}{\sqrt{2}} \\
& =\frac{\left[\ldots 2 p_{x^{\prime}}(1) 2 p_{y^{\prime}}(2)+\ldots 2 p_{y^{\prime}}(1) 2 p_{x^{\prime}}(2)\right][\alpha(1) \beta(2)-\beta(1) \alpha(2)]}{2}
\end{aligned}
$$

where

$$
2 p_{x^{\prime}}=\left(\frac{2 p_{x}+2 p_{y}}{\sqrt{2}}\right)
$$

and

$$
2 p_{y^{\prime}}=\left(\frac{2 p_{x}-2 p_{y}}{\sqrt{2}}\right)
$$




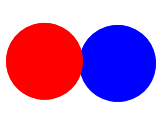

$2 p_{x}$

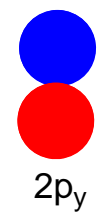

(a)

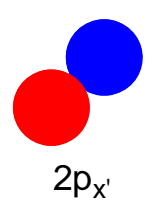

(b)

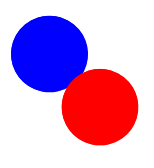

$2 p_{y^{\prime}}$

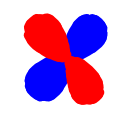

$2 p_{y} \times 2 p_{y}$

(c)

Figure 2. (a) One possible choice of the degenerate $2 p$ AOs in $\mathrm{HN}$, when the $\mathrm{N}-\mathrm{H}$ bond lies along the $\mathrm{z}$ axis. (b) Another possible choice of the degenerate $2 \mathrm{p}$ AOs in $\mathrm{HN}$, differing by a $45^{\circ}$ rotation of the $\mathrm{x}$ and y coordinate axes, (c) Schematic depiction of the overlap between the $2 p_{\mathrm{x}}$ and $2 \mathrm{p}_{\mathrm{y}}$ AOs. As shown in eq. 15 , if $\Psi_{\mathrm{TC}}$ is written in terms of the $2 \mathrm{p}_{\mathrm{x}}$ and $2 \mathrm{p}_{\mathrm{y}}$ AOs that are depicted in (a), the GVB orbitals that are derived from $\Psi_{\mathrm{TC}}$ are the $2 \mathrm{p}_{\mathrm{x}^{\prime}}$ and $2 \mathrm{p}_{\mathrm{y}^{\prime}}$ AOs that are depicted in (b).

As shown in Fig. 2b, the primed GVB orbitals for $\mathrm{HN}$ are really the same as the unprimed MOs, but just rotated by $45^{\circ}$. Since the spatial orientation of the $\mathrm{x}$ and $\mathrm{y}$ coordinate axes in $\mathrm{HN}$ is purely arbitrary, in $\mathrm{HN}$ the energies of the "open-shell" singlet wave function, $\Psi_{\mathrm{s} 0}$, in eq. 14 and the "closed-shell" singlet wave function, $\Psi_{\mathrm{TC}}\left(=\Psi_{\mathrm{S} 0^{\prime}}\right)$ in eq. 15 must also the same. Therefore, in HN symmetry makes $\left(\mathrm{J}_{\mathrm{xx}}+\mathrm{J}_{\mathrm{yy}}\right) / 2-\mathrm{J}_{\mathrm{xy}}=2 \mathrm{~K}_{\mathrm{xy}}$.

The HN diradical does, in fact, have a ${ }^{1} \Delta$ state that is doubly degenerate. The MOs that have angular momentum quantum numbers of $\mathrm{L}_{\mathrm{z}}= \pm 1$ are complex MOs of the form, $\psi_{\mathrm{x}} \pm \mathrm{i} \psi_{\mathrm{y}}$. The two, degenerate $\mathrm{L}_{\mathrm{z}}= \pm 2$ components of the ${ }^{1} \Delta$ state of $\mathrm{HN}$ are, in fact, linear combinations of $\Psi_{\mathrm{S} 0}$ and $\Psi_{\mathrm{TC}}$.

2.4.2. GVB MOs of Square Cyclobutadiene. It is not always the case that $\Psi_{\mathrm{S} 0}$ and $\Psi_{\mathrm{TC}}$ have the same energies. An example of a diradical in which the "open-shell" singlet wave function and the TC, "closed-shell" singlet wave function have different energies is provided by square cyclobutadiene (CBD). Transformation of the TC, "closed-shell", singlet wave function to an open-shell singlet wave function, written in terms of GVB orbitals, is a convenient way of demonstrating that $\mathrm{E}\left(\Psi_{\mathrm{S} 0}\right) \neq \mathrm{E}\left(\Psi_{\mathrm{TC}}\right)$ in square cyclobutadiene, which is the transition structure that connects the two, rectangular, equilibrium geometries of the singlet state. The highest occupied (HO)MO and lowest unoccupied (LU)MO at each of these latter two geometries are shown in Figure 3a. For the rectangular geometry that has the two long $\mathrm{C}-\mathrm{C}$ bonds parallel to the $\mathrm{x}$ axis, $\psi_{\mathrm{x}}$ is lower in energy than $\psi_{\mathrm{y}}$. For the rectangular geometry with the two long $\mathrm{C}-\mathrm{C}$ bonds parallel to the $y$ axis, the ordering of $\psi_{\mathrm{x}}$ and $\psi_{\mathrm{y}}$ is reversed. However, at the square geometry of the transition structure, symmetry makes $\psi_{\mathrm{x}}$ and $\psi_{\mathrm{y}}$ nonbonding and degenerate in energy.

A wave function that has the flexibility to describe the potential surface that connects the rectangular equilibrium geometries of $\mathrm{CBD}$ is the two-configuration, singlet wave function,

$$
\Psi_{T C}=c_{x}^{2}\left|\ldots \psi_{x}^{2}\right\rangle-c_{y}^{2}\left|\ldots \psi_{y}^{2}\right\rangle
$$


for which the GVB orbitals are

$$
\psi_{x^{\prime}}=c_{x} \psi_{x}+c_{y} \psi_{y}
$$

and

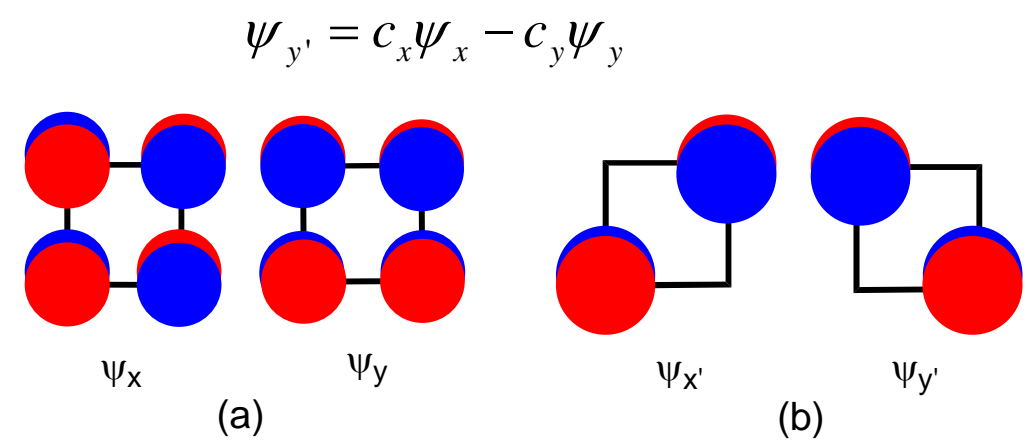

Figure 3. Two possible choices, differing by a $45^{\circ}$ rotation of the $\mathrm{x}$ and $y$ coordinate axes, of the degenerate nonbonding MOs in square

cyclobutadiene. The primed MOs, $\psi_{\mathrm{x}^{\prime}}$ and $\psi_{\mathrm{y}^{\prime}}$ in (b) can be considered to be the GVB orbitals, derived from $\Psi_{\mathrm{TC}}$, when $\Psi_{\mathrm{TC}}$ is written in terms of the MOs $\psi_{\mathrm{x}}$ and $\psi_{\mathrm{y}}$ in (a).

At rectangular geometries, where $\psi_{\mathrm{x}}$ is lower in energy than $\psi_{\mathrm{y}},\left|\mathbf{c}_{\mathrm{x}}\right|>\left|\mathrm{c}_{\mathrm{y}}\right|$; and at rectangular geometries, where $\psi_{\mathrm{x}}$ is higher in energy than $\psi_{\mathrm{y}},\left|\mathbf{c}_{\mathrm{x}}\right|<\left|\mathrm{c}_{\mathrm{y}}\right|$. At square geometries, $\mathrm{c}_{\mathrm{x}}$ $=c_{y}$. Therefore, the GVB orbitals, $\psi_{x^{\prime}}$ and $\psi_{y^{\prime}}$, for square CBD are the sum and difference of the MOs, $\psi_{\mathrm{x}}$ and $\psi_{\mathrm{y}}$, that have the correct symmetry for describing rectangular CBD. The GVB orbitals, $\psi_{\mathrm{x}^{\prime}}$ and $\psi_{\mathrm{y}^{\prime}}$, for square CBD are shown in Fig. $3 \mathrm{~b}$.

Comparison of the MOs in Figs. 3a and $3 \mathrm{~b}$ show that, like the unprimed and primed MOs for $\mathrm{HN}$ in Fig. 2, the MOs in Fig. 3 are related by a $45^{\circ}$ rotation of the $\mathrm{x}$ and $\mathrm{y}$ axes. However, unlike the case in $\mathrm{HN}$, rotation by $45^{\circ}$ is not a symmetry operation for square $\mathrm{CBD}$. Therefore, the degenerate MOs in Figs. $3 \mathrm{a}$ and $3 \mathrm{~b}$ are not the same. The unprimed MOs, $\psi_{\mathrm{x}}$ and $\psi_{\mathrm{y}}$, in Fig. 3a have nodal planes that pass through the $\mathrm{C}-\mathrm{C}$ bonds of $\mathrm{CBD}$; whereas, the primed GVB orbitals, $\psi_{\mathrm{x}^{\prime}}$ and $\psi_{\mathrm{y}^{\prime}}$ in Fig $3 \mathrm{~b}$ have nodal planes that pass through the carbons.

Since the unprimed, $\psi_{\mathrm{x}}$ and $\psi_{\mathrm{y}}$, in Fig. 3a, have atoms in common, $\mathrm{K}_{\mathrm{xy}}$ is large. Therefore, the open-shell singlet wave function, in which one electron occupies $\psi_{\mathrm{x}}$ and another electron of opposite spin occupies $\psi_{\mathrm{y}}$, has a very high Coulombic repulsion energy. In contrast, the GVB orbitals, $\psi_{\mathrm{x}^{\prime}}$ and $\psi_{\mathrm{y}^{\prime}}$, in Fig. $3 \mathrm{~b}$ are disjoint; i.e., they have no atoms in common. Therefore, $\mathrm{K}_{\mathrm{xy}} \approx$ 0 ; so the "closed-shell", TC wave function, from which the GVB MOs in Fig. 3b are derived, has a much lower Coulombic repulsion energy than the "open-shell" wave function, constructed from the MOs in Fig. 3a.

\subsubsection{Dynamic Spin Polarization and the Predicted Violation of Hund's Rule in} Square CBD. Since the GVB MOs in Fig. 3b are disjoint, the lowest singlet state of square $\mathrm{CBD}$, in which one electron occupies $\psi_{\mathrm{x}^{\prime}}$ and the other $\psi_{\mathrm{y}^{\prime}}$, has approximately the same energy as the triplet state. With an electron of $\alpha$ spin in $\psi_{x^{\prime}}$ and an electron of $\beta$ spin in $\psi_{y^{\prime}}$, the asymmetric distribution of spin in the singlet GVB orbitals favors the $\alpha$ and $\beta$ spin electrons in the lowest $\pi$ MO having slightly different wave functions. In order to minimize the Coulombic repulsion between electrons of opposite spin, the $\alpha$ spin electron in the lowest $\pi$ MO tends to become 
localized at the same pair of carbons as the $\alpha$ spin electron in $\psi_{x^{\prime}}$, and the $\beta$ spin electron in the lowest $\pi \mathrm{MO}$ tends to become localized at the same pair of carbons as the $\beta$ spin electron in $\psi_{y^{\prime}}$.

Of course, $\mid \ldots \psi_{x^{\prime}}{ }^{\alpha} \psi_{y^{\prime}}>$ represents only half of the singlet wave function for square CBD. Therefore, in the lowest singlet state, polarization of the electrons in the bonding $\pi \mathrm{MO}$ of square CBD must be dynamic, rather than static. Nevertheless, a physical model in which, in order to minimize the Coulombic repulsion between electrons of opposite spin, each of the electrons in the lowest $\pi \mathrm{MO}$ tends to become more localized at the pair of carbons at which an electron of the same spin is localized in the GVB MOs, provides a reasonable description of the singlet wave function for square $\mathrm{CBD}{ }^{16,17}$

In contrast to the case in singlet $\mathrm{CBD}$, triplet $\mathrm{CBD}$ has a uniform distribution of spin in the nonbonding $\pi$ MOs. Consequently, in the triplet, dynamic spin polarization cannot reduce the Coulombic repulsion between the electrons in the nonbonding MOs and the electron of opposite spin in the bonding $\pi \mathrm{MO}$. As a result of the selective stabilization of the singlet by dynamic spin polarization, the fact that $\mathrm{K}_{\mathrm{xy}} \approx 0$ in square $\mathrm{CBD}$ results in the singlet being computed to be the ground state of square $\mathrm{CBD} .^{16-22}$

\subsection{Disjoint and Non-Disjoint MOs and the Qualitative Prediction of $\Delta E_{\mathrm{ST}}$ in} Alternant Hydrocarbon Diradicals. The foregoing discussion leads to a simple procedure for qualitatively predicting the ground state of a diradical. (a) Find the degenerate triplet nonbonding MOs (NBMOs) of highest symmetry. Since these NBMOs will usually be delocalized, they will, in general, have atoms in common. (b) Form the GVB NBMOs, which are the sum and difference of the delocalized NBMOs. (c) If either the original NBMOs or the GVB orbitals derived from them is disjoint, dynamic spin polarization is likely to make the singlet the ground state. (d) If neither the triplet NBMOs nor the singlet GVB NBMOs is disjoint, the ground state of the diradical is the triplet; and the optimal NBMOs for $\Psi_{\mathrm{s} 0}$ in eq. 2 and the optimal GVB MOs for $\Psi_{\mathrm{TC}}$ in eq. 10 are both likely to differ from the NBMOs for the triplet.

If a diradical is an alternant hydrocarbon (AH), determining whether or not its NBMOs or GVB orbitals are disjoint is straightforward. ${ }^{2,23} \mathrm{An} \mathrm{AH}$ is a conjugated hydrocarbon in which the carbons can be divided into two sets, traditionally called the "starred" and "unstarred" sets, such that starred carbons have only unstarred carbons as nearest neighbors and vice versa. ${ }^{24,25}$ Figure 4 shows that CBD and trimethylenemethane (TMM) are both AHs.

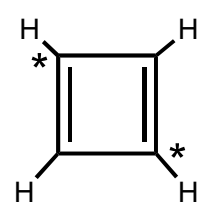

CBD

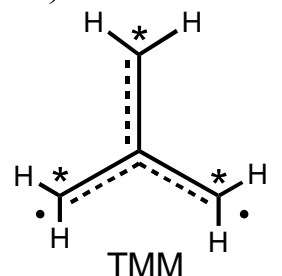

Figure 4. Starred and unstarred carbons in two alternant hydrocarbons, CBD and TMM.

Although CBD and TMM are both AHs, they differ in one important respect. In CBD the number of starred carbons $\left(\mathrm{n}_{\mathrm{s}}\right)$ is equal to the number of unstarred carbons $\left(\mathrm{n}_{\mathrm{u}}\right)$, but in TMM $\mathrm{n}_{\mathrm{s}}-$ $\mathrm{n}_{\mathrm{u}}=2$. It can be proven ${ }^{2,26}$ that in a diradical in which $\mathrm{n}_{\mathrm{s}}=\mathrm{n}_{\mathrm{u}}$, the NBMOs can be chosen to be disjoint, with one NBMO confined to the starred set of atoms and the other to the unstarred set. 
We have already seen that the GVB orbitals for CBD in Fig. $3 b$ can be chosen so that this is the case.

In contrast, when $\mathrm{n}_{\mathrm{s}}-\mathrm{n}_{\mathrm{u}}=2$, both NBMOs are localized at only the more numerous set of atoms, which by convention is chosen to be the starred set. Figure 5a shows the NBMOs for TMM, and they are obviously non-disjoint. Consequently, just from the fact that $n_{s}-n_{u}=2$ in TMM, it can be predicted that (a) TMM has a triplet ground state and (b) that the optimal NBMOs for the lowest singlet states differ from those of the triplet state. ${ }^{2}$

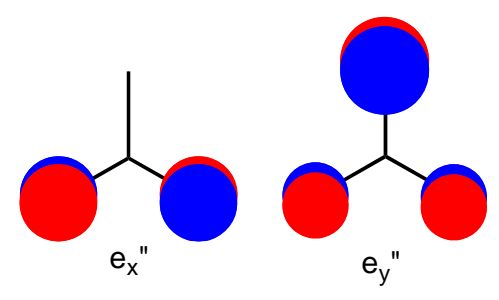

(a)

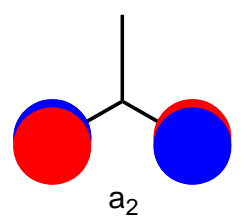

(b)

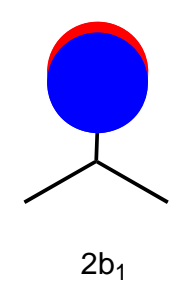

Figure 5. Nonbonding MOs of (a) ${ }^{3} \mathrm{~A}_{2}$ ' TMM and (b) ${ }^{1} \mathrm{~B}_{2} \mathrm{TMM}$.

In fact, the optimal NBMOs for the "open-shell" singlet state $\left({ }^{1} \mathrm{~B}_{2}\right)$ of TMM, in which each of the NBMOs is occupied by one electron, differ from the degenerate pair of $\mathrm{e}^{\text {" NBMOs }}$ for the ${ }^{3} \mathrm{~A}_{2}$ ' state. In order to minimize the regions of space in which the NBMOs overlap in the ${ }^{1} \mathrm{~B}_{2}$ state, the optimal $2 \mathrm{~b}_{1}$ NBMO for the singlet, which is shown in Fig. 5b, is localized to a carbon atom where the $\mathrm{a}_{2}$ NBMO has a node. The localization of the NBMOs of the singlet to different sets of carbons means that the Coulomb repulsion between the electrons of opposite spin in them is actually about the same as that between the electrons of the same spin in the degenerate $e_{x} "$ and $e_{y} "$ NBMOs for the triplet, which are shown in Fig. 5a.

However, the reduction in Coulombic repulsion between the electrons of opposite spin in the singlet has an energetic cost. The "open-shell" wave function for singlet TMM can be accurately be described as the wave function for an allyl radical, with an electron of spin opposite to that of the electron in the allyl NBMO localized at the unique carbon atom. In Hückel theory, the energy of the pair of electrons in the bonding MO of allyl is $2 \alpha+2 \sqrt{ } 2 \beta$; whereas the energy of the pair of electrons in the bonding MO of TMM is $2 \alpha+2 \sqrt{3} \beta$. Thus, even within a simple Hückel model, the modification in the singlet of the NBMOs for the triplet, depicted in Figure 5, results in the pair of $\pi$ bonding electrons in the singlet having an energy that is $0.6|\beta|$ higher than the energy of the pair of $\pi$ bonding electrons in the triplet.

There is another effect that raises the energy of the planar singlet. If there is an $\alpha$ spin electron in the nonbonding orbital of an allylic radical, spin polarization results in $\beta$ spin appearing at the central carbon. ${ }^{27}$ Thus, in singlet trimethylene, there are electrons of the same spin in the $2 p-\pi$ AOs at the central and the unique peripheral carbons. This produces an antibonding $\pi$ interaction between these two $2 p$ AOs. Consequently, unlike the triplet state, the preferred geometry of the lowest singlet state of TMM is predicted not to be planar, but to have the unique $\mathrm{CH}_{2}$ group twisted out of conjugation. ${ }^{1}$ This prediction is consistent with not only the 
results of $a b$ initio calculations, ${ }^{2,28,29}$ but also with results on the stereochemistry of the thermal methylenecyclopropane rearrangement, in which singlet TMM serves as an intermediate. ${ }^{30-33}$

As will be discussed in Section 4.3, the fact that the lowest singlet state of TMM has an energy minimum at a geometry with one $\mathrm{CH}_{2}$ group twisted out of conjugation, means that this geometry cannot be accessed by negative ion photoelectron spectroscopy (NIPES). NIPES involves photochemically detaching an electron from a radical anion, and the radical anion of TMM (TMM $\bullet^{-}$) has a planar equilibrium geometry. The Franck-Condon factor for going from the planar geometry of TMM $\bullet^{-}$to the twisted energy minimum for singlet TMM is vanishingly small.

\section{Negative Ion Photoelectron Spectroscopy and measurement of $\Delta E_{\mathrm{ST}}$ in Diradicals}

In negative ion photoelectron spectroscopy (NIPES), a beam of mass-selected anions is intersected by a fixed frequency laser beam. In the interaction between them, an electron is ejected from the molecular anion in the photodetachment process. In order to obtain the photoelectron spectrum, the kinetic energies of the photodetached electrons are measured. Energy conservation requires that the kinetic energy of the photoelectron be equal to the photon energy less the energy difference between the initial state of the anion and the final state of the neutral molecule. The final state of the neutral molecule may be the ground state or vibrationally or electronically excited states. Thus measurement of the electron kinetic energy yields a vibrational and electronic spectrum of the transition between the anion and the neutral.

Negative ion photoelectron spectroscopy has several strengths, relative to other spectroscopic methods. First, because the ions can be mass selected, the signal carrier or the photoelectron spectrum is unambiguously established. Second, NIPES is a general method for obtaining the vibrational and electronic spectra of neutral molecules, including reactive, shortlived, and radical species. Finally, because an electron is removed in the photodetachment process, the selection rule for electron spin is $\Delta S= \pm 1 / 2$, and neutral electronic states of different spin multiplicity are readily seen in the photoelectron spectrum. For the purpose of the research described in this Perspective, it is very fortunate that a doublet radical anion yields both singlet and triplet states of the neutral in the photoelectron spectrum, so that the singlet-triplet energy splitting can be measured directly.

Accordingly, the principal components of a negative ion photoelectron spectrometer are the ion beam source, mass analyzer, the laser source and the electron energy analyzer. Ions which have been mass selected and focused into a beam are crossed at 90 degrees with the high intensity continuous laser beam. Photoelectrons are collected in the direction perpendicular to the ion and laser beams and are sent through an electrostatic energy analyzer, which disperses the electrons according to kinetic energy. The photoelectron intensity is monitored as the analyzer is scanned over electron kinetic energies.

The first negative ion photoelectron spectrometer was constructed in the early 1970s by John Hall and co-workers in JILA at the University of Colorado. This early instrument required a high intensity discharge anion source, a home-built argon-ion visible laser, and employed a hemispherical electrostatic energy analyzer with $50 \mathrm{meV}$ energy resolution. ${ }^{34,35}$ It is essentially this instrument, with modest improvements, that was utilized in the Lineberger group through 1977. That interval included the first applications to real chemical problems, including measurements of a number of organic spin-state splittings, ${ }^{36-38}$ and the first investigation ${ }^{39}$ of $\mathrm{CH}_{2}{ }^{-}$, discussed in Section 4.2. 
In 1983, a sequence of major NIPES instrument improvements was completed, affording an order of magnitude increase in electron energy resolution to $\sim 5 \mathrm{meV}$, and a near 1000 -fold increase in sensitivity. These two developments in turn allowed the electrical discharge ion source to be replaced with a much lower intensity, but far better characterized, flowing afterglow, ion-molecule reactor as the ion source. This change afforded both rational ion formation and ion cooling prior to photodetachment. The ability to carry out rational gas-phase syntheses of complex anions at defined temperatures enabled virtually all of the anion spectroscopy described in this article. The first project to be undertaken with this completely new apparatus was a re-investigation ${ }^{40}$ of the controversial singlet-triplet splitting in $\mathrm{CH}_{2}$, as described in section 4.2 .

The full analysis of a photoelectron spectrum provides a great deal of additional information beyond the electronic state energies that are the focus of this Perspective. A full vibrational state simulation yields important information on energy levels, anharmonicities, mode coupling, and electronic state couplings. Such analyses have been carried out for all of the photoelectron spectra reported here, and add important information concerning the diradicals. However, when the geometry differences between anion and neutral electronic state(s) are modest, electronic state splittings can be determined directly from the experimental data, without the necessity of a full vibrational analysis. For this reason, we provide only a limited a limited discussion of the additional issues that arise when geometry changes are significant. Triplet methylene and, especially, triplet dichloromethylene represent cases where further reliance on theory for electronic state splittings is critical. This issue is discussed in more detail in Section 4.2, which describes experiment and calculations for these two diradicals.

\section{Calculations and Measurements of $\Delta E_{\mathrm{ST}}$ in Some Diradicals.}

In this section we describe briefly the calculation and measurement of $\Delta E_{\mathrm{ST}}$ in a representative collection of diradicals. A recent review by Wenthold ${ }^{41}$ gives a much more complete compilation of the values of $\Delta E_{\mathrm{ST}}$ that have been measured by NIPES, largely by WCL and his collaborators.

4.1. Nitrenes $-\mathbf{H N}, \mathrm{H}_{3} \mathrm{CN}$, and $\mathrm{PhN}$. One of the first measurements of the singlettriplet energy difference in a diradical by NIPES was made in $1976^{36}$ on HN. The photoelectron spectrum consisted of two well -separated peaks corresponding to $\mathrm{v}=0$ of the singlet and triplet states. Due to the three-fold degeneracy of the $S_{Z}=1,0$, and -1 components of the triplet, the intensity of the peak for the ${ }^{3} \Sigma^{-}$state was roughly a factor of $50 \%$ greater than that for the doubly degenerate ${ }^{1} \Delta$ state. From the NIPE spectrum, a value of $\Delta E_{\mathrm{ST}}=36.5 \pm 0.4 \mathrm{kcal}$ was obtained, with the positive sign indicating that the triplet state is lower in energy than the singlet state. This result was in excellent agreement with the more precise spectroscopic value ${ }^{42}$ obtained one year earlier by Zetzsch and Stahl.

As can be seen in Fig. 2c, there are four regions of space in which the nitrogen $2 p_{x}$ and $2 p_{y}$ AOs orbitals overlap. The correlation between the two electrons of the same spin, which occupy these AOs in the ${ }^{3} \Sigma^{-}$state, prevents these electrons from simultaneously appearing in the same one of these four regions. In contrast, in the ${ }^{1} \Delta$ state, the pair of electrons that occupy these two AO are anti-correlated, so that the probability of these electrons appearing in the same one 
of these four regions is actually higher than would be computed from the product of the squares of the $2 p_{x}$ and $2 p_{y}$ AOs. Consequently, because the $2 p_{x}$ and $2 p_{y}$ AOs on nitrogen have regions of space in common, the triplet state $\left({ }^{3} \Sigma^{-}\right)$is much lower than the lowest singlet state $\left({ }^{1} \Delta\right)$ in HN.

In $\mathrm{H}_{3} \mathrm{CN}$ delocalization of the electrons in the degenerate pair of $2 \mathrm{p}$ AOs into combinations of the methyl C-H orbitals of the same symmetry reduces the Coulombic repulsion in the lowest singlet state from that in $\mathrm{HN}$. Consequently, the singlet-triplet energy difference in $\mathrm{H}_{3} \mathrm{CN}$, measured by NIPES, is $\Delta E_{\mathrm{ST}}=31.2 \pm 0.3 \mathrm{kcal} / \mathrm{mol}$, ${ }^{43}$ which is $5.3 \mathrm{kcal} / \mathrm{mol}$ smaller than $\Delta E_{\mathrm{ST}}$ in $\mathrm{HN}$.

Since singlet $\mathrm{H}_{3} \mathrm{CN}$ can undergo a very exothermic rearrangement to $\mathrm{H}_{2} \mathrm{C}=\mathrm{NH}$, concern was expressed that singlet $\mathrm{H}_{3} \mathrm{CN}$ might not be an energy minimum. ${ }^{43}$ However, subsequent $a b$ inito calculations found that there is, in fact, a barrier to this reaction; so the singlet state of $\mathrm{CH}_{3} \mathrm{~N}$ really does appear to be an energy minimum. ${ }^{44}$

In the case of $\mathrm{PhN}$, two sets of ab initio calculations of $\Delta E_{\mathrm{ST}}$, performed by two different research groups, ${ }^{45,46}$ and experimental measurements of $\Delta E_{\mathrm{ST}}{ }^{47,48}$ appeared almost simultaneously. Both the calculations and experiments found $\Delta E_{\mathrm{ST}} \approx 18 \mathrm{kcal} / \mathrm{mol}$, a considerably smaller value than in either $\mathrm{HN}$ or $\mathrm{H}_{3} \mathrm{CN}$. The most recent investigation ${ }^{49}$ of $\mathrm{PhN}^{-}$corrects a misassignment in the Travers study ${ }^{47}$, giving an even smaller value for $\Delta E_{\mathrm{ST}}$ in $\mathrm{PhN}, \Delta E_{\mathrm{ST}}=$ $14.8 \pm 0.5 \mathrm{kcal} / \mathrm{mol}$. This latter result is consistent with the most recent calculation, ${ }^{50}$ which obtained $\Delta \mathrm{E}_{\mathrm{ST}}=15.9 \mathrm{kcal} / \mathrm{mol}$.

The calculations provided something that the experiments could not - an explanation of the reason for the $>50 \%$ reduction in the value of $\Delta E_{\mathrm{ST}}$ in $\mathrm{PhN}$ from the value in $\mathrm{HN}{ }^{46}$ Unlike the case in $\mathrm{HN}$ or $\mathrm{H}_{3} \mathrm{CN}$, in $\mathrm{PhN}$ the two lowest singlet states are not degenerate by symmetry. In the "open-shell", ${ }^{1} \mathrm{~A}_{2}$ state, one electron occupies a $\sigma$ orbital, which is largely localized on nitrogen, and another electron of opposite spin occupies a $\pi$ MO that extends over the benzene ring. In the "closed-shell", ${ }^{1} \mathrm{~A}_{1}$ state, the wave function has the form of eq. 18 and contains two configurations, in each of which the two nonbonding electrons occupy the same MO, either the $\sigma$ on $\mathrm{N}$ or a $\pi$ NBMO.

Since the two configurations in ${ }^{1} \mathrm{~A}_{1}$ need not have the same weight, in this singlet state the pair of electrons can preferentially occupy the MO that has lower energy. Therefore one might have expected the lower energy singlet state in $\mathrm{PhN}$ would be ${ }^{1} \mathrm{~A}_{1}$. However, that was not found to be the case. The energy difference between ${ }^{3} \mathrm{~A}_{2}$ and ${ }^{1} \mathrm{~A}_{1}$ was calculated to be ca. 30 $\mathrm{kcal} / \mathrm{mol}^{45,46,50}$ about the same size as the singlet-triplet splitting in $\mathrm{H}_{3} \mathrm{CN} .{ }^{43}$ The much lower energy singlet state was the open-shell ${ }^{1} \mathrm{~A}_{2}$ state. ${ }^{45,46,50}$

The calculations not only identified which is the lower energy of the two singlet states in $\mathrm{PhN}$ but also why, relative to the triplet, this state is much lower in energy in $\mathrm{PhN}$. than in $\mathrm{HN}$. The clue to the answer was found in the bond lengths computed for the three low-lying states of $\mathrm{PhN}{ }^{46}$ One set of the calculated bond lengths is reproduced in Fig. $6 .{ }^{50}$ 

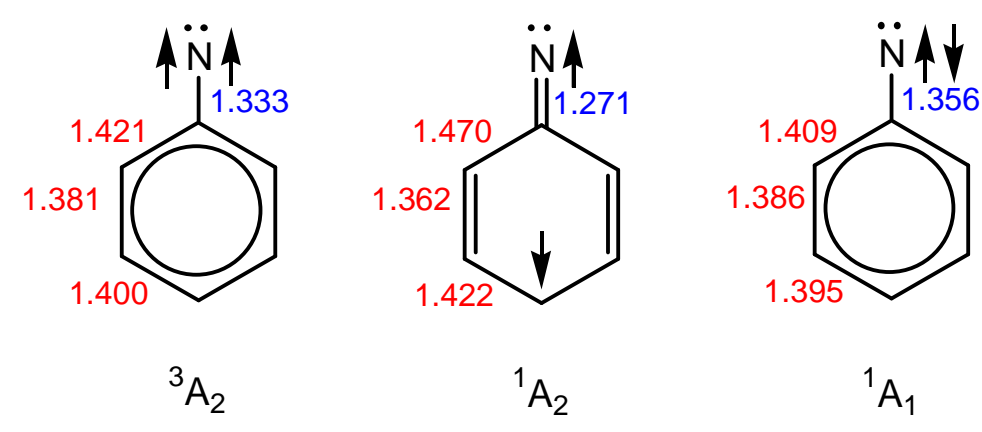

Figure 6. Calculated bond lengths in $\AA$ in the three lowest states of $\mathrm{PhN} .^{47}$

All three states have optimized bond lengths that are different from each other. The alternation of the $\mathrm{C}-\mathrm{C}$ bond lengths of the benzene ring in the ${ }^{3} \mathrm{~A}_{2}$ state suggests a modest amount of delocalization of the $\pi$ electron, nominally shown as being localized on nitrogen in Fig. 6, into the benzene ring. However, in the ${ }^{1} \mathrm{~A}_{2}$ state there is a much larger amount of alternation of the $\mathrm{C}-\mathrm{C}$ bond lengths in the benzene ring; and the $\mathrm{C}-\mathrm{N}$ bond length in the open-shell singlet is much shorter than in the triplet. Therefore, it is clear that the nonbonding $\pi$ electron must be largely delocalized from nitrogen and into the benzene ring in the ${ }^{1} \mathrm{~A}_{2}$ state. Finally, there is even less alternation of the $\mathrm{C}-\mathrm{C}$ bond lengths in the benzene ring in the ${ }^{1} \mathrm{~A}_{1}$ state than in the ${ }^{3} \mathrm{~A}_{2}$ state, and the $\mathrm{C}-\mathrm{N}$ bond length in the closed-shell singlet is even longer than in the triplet.

The fact that the optimized bond lengths in the three lowest states of $\mathrm{PhN}$ are so different means that a different set of MOs must be optimal for each of the states. Why are the MOs for the triplet not optimal for the singlets too? One way to answer this question is to note that $\mathrm{K}_{\mathrm{ij}}$ enters the expression in eq 6 for the energy of the open-shell singlet with a positive sign. Localizing the electrons of opposite spin to different regions of space - the nonbonding nitrogen $\sigma$ orbital and the $\pi$ system of the benzene ring - minimizes the size of $K_{i j}$ in the ${ }^{1} A_{2}$ state. In contrast, $\mathrm{K}_{\mathrm{ij}}$ enters the expression in eq 8 for the energy of the closed-shell singlet with a negative sign; and localizing both of the NBMOs on nitrogen maximizes the size of $\mathrm{K}_{\mathrm{ij}}$.

Since the optimal NBMOs for the ${ }^{1} \mathrm{~A}_{1}$ state of $\mathrm{PhN}$ are really not very different from those for the singlet state of $\mathrm{H}_{3} \mathrm{CN}$, it is understandable that the energy difference between ${ }^{1} \mathrm{~A}_{1}$ and ${ }^{3} \mathrm{~A}_{2}$ in $\mathrm{PhN}$ is calculated to be about the same as is in $\mathrm{H}_{3} \mathrm{CN}$. However, in contrast to the case in the singlet state of $\mathrm{H}_{3} \mathrm{CN}$, in the ${ }^{1} \mathrm{~A}_{2}$ state of $\mathrm{PhN}$ the nonbonding electrons of opposite spin are confined to different regions of space. Since their mutual Coulombic repulsion is, therefore, much lower than in $\mathrm{H}_{3} \mathrm{CN}$, the singlet-triplet splitting in $\mathrm{PhN}$ is only about half of that in $\mathrm{H}_{3} \mathrm{CN}$.

Understanding the nature of the open-shell singlet wave function for the ${ }^{1} \mathrm{~A}_{2}$ state of $\mathrm{PhN}$ provided tremendous insight into the chemistry of the lowest singlet state of $\mathrm{PhN}$ and into the large differences between the chemistries of the lowest singlet states of $\mathrm{PhN}$ and the isoelectronic $\mathrm{PhCH}{ }^{51,52}$ The fact that calculations could predict accurately the experimentally measured value of $\Delta E_{\mathrm{ST}}$ in $\mathrm{PhN}$ gave credence to the calculated description of the lowest singlet state of $\mathrm{PhN}$ as having an unpaired electron localized in a $\sigma$ orbital on $\mathrm{N}$ and an electron of opposite spin largely localized in the $\pi$ system of the benzene ring. 
4.2. Methylene $\left(\mathrm{CH}_{2}\right)$. The very interesting story of the calculations and measurements of $\Delta E_{\mathrm{ST}}$ in $\mathrm{CH}_{2}$ has been retold in several excellent reviews. ${ }^{53-56}$ Therefore, we will recapitulate the story here only briefly. Extensive references to the original literature can be found in the reviews mentioned above.

In analogy to the case in $\mathrm{HN}$, at linear geometries the nonbonding $\mathrm{MOs}$ of $\mathrm{CH}_{2}$ are a pair of $2 \mathrm{p} \mathrm{AOs}$. Consequently, as in $\mathrm{HN}$, in linear $\mathrm{CH}_{2}$ the triplet can be predicted to be the ground state, with the lowest singlet state being doubly degenerate.

However, replacing the lone pair of electrons, which occupies a nominally sp hybridized $\mathrm{AO}$ in $\mathrm{NH}$, with an sp $\mathrm{CH}$ bond has an important consequence. Although bending is undefined in $\mathrm{HN}$, bent geometries are possible $\mathrm{CH}_{2}$. Bending transforms the $2 \mathrm{p} \mathrm{AO}$ on carbon, which lies in the plane in which bending, occurs, into a weakly $\mathrm{C}-\mathrm{H}$ bonding hybrid. In the lowest singlet state the pair of electrons that occupy the nonbonding $2 \mathrm{p} \mathrm{AOs}$ in linear $\mathrm{CH}_{2}$ both tend to occupy the orbital that is stabilized by bending. The degeneracy of the lowest singlet state of linear $\mathrm{CH}_{2}$ is thus lifted, and it is easy to predict that the lowest singlet state of $\mathrm{CH}_{2}$ will be a highly bent, closed-shell, ${ }^{1} \mathrm{~A}_{1}$ state, rather than an open-shell ${ }^{1} \mathrm{~B}_{1}$ state, in which only one electron occupies the orbital that is stabilized by bending.

The triplet state also has only one electron in the MO that is stabilized by bending; so the ${ }^{3} \mathrm{~B}_{1}$ should have a less bent geometry than ${ }^{1} \mathrm{~A}_{1}$. Although a spectroscopic study by Herzberg initially indicated that the triplet state might have a linear geometry, ${ }^{57}$ several calculations indicated that triplet $\mathrm{CH}_{2}$ was bent with a bond angle of ca. $135^{\circ} .{ }^{53-56,58-60} \mathrm{An}$ EPR study of triplet $\mathrm{CH}_{2}$ by Wasserman and coworkers confirmed that the calculations were, indeed, correct. ${ }^{61}$

At a linear geometry, the triplet state of $\mathrm{CH}_{2}$ is lower in energy than the degenerate singlet by $2 \mathrm{~K}_{\mathrm{xy}}$. However, on bending, the ${ }^{1} \mathrm{~A}_{1}$ component of the linear singlet state is stabilized by more than the ${ }^{3} \mathrm{~B}_{1}$ state. Consequently, without actually doing electronic structure calculations or spectroscopic experiments, there is no way to determine which of these two states is lower in energy at their respective equilibrium geometries.

Indeed, although calculations and experiments both find the triplet to be the ground state of $\mathrm{CH}_{2},{ }^{53-56}$ moving down a row of the periodic table, the ground state of $\mathrm{SiH}_{2}$ was calculated to be a singlet, ${ }^{62}$ and a low resolution NIPES experiment ${ }^{38}$ appeared to agree, reporting that the triplet state was less than $14 \mathrm{kcal} / \mathrm{mol}$ higher than the singlet.

By the mid-1970s, both $a b$ initio calculations and indirect experiments seemed to be converging on a singlet-triplet energy difference in $\mathrm{CH}_{2}$ on the order of $\Delta E_{\mathrm{ST}} \approx 10 \mathrm{kcal} / \mathrm{mol}{ }^{62-69}$ Therefore, when the first NIPE spectrum of $\mathrm{CH}_{2}{ }^{-{ }^{-}}$was published, it came as a shock that the energy difference between the first peak for formation of ${ }^{3} \mathrm{~B}_{1}$ and that for formation of ${ }^{1} \mathrm{~A}_{1}$ was reported to be $19.5 \pm 0.7 \mathrm{kcal} / \mathrm{mol}^{39}$ The NIPE spectrum is reproduced in Fig. 7. 


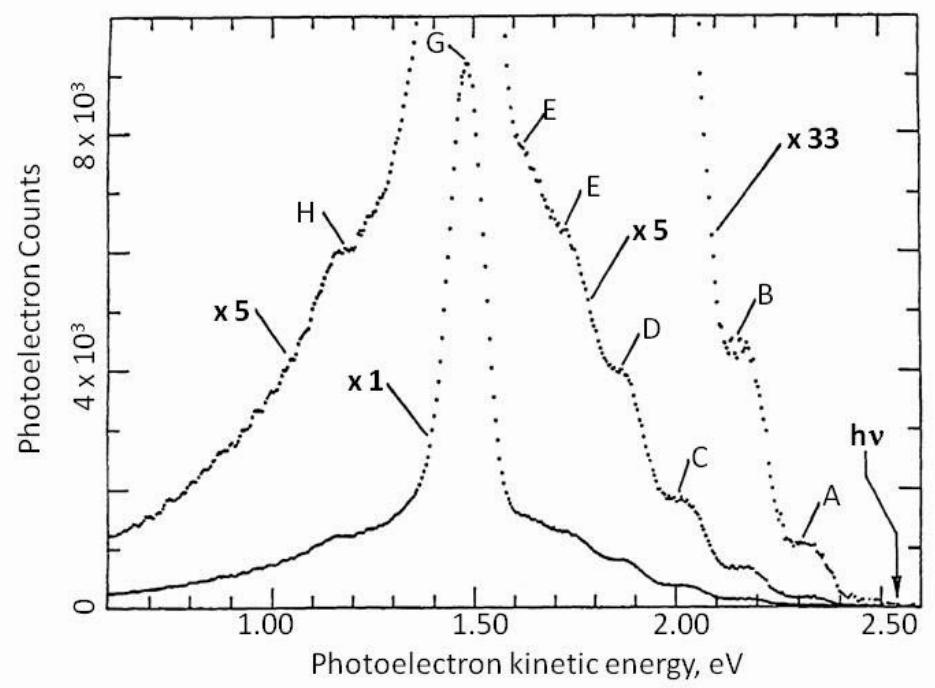

Figure 7. Photoelectron spectrum of $\mathrm{CH}_{2}{ }^{--}$obtained in $1976^{39}$ using the original instrument with an electron energy resolution of $400 \mathrm{~cm}^{-1}$ and an electrical discharge ion source that provided no control of anion temperature. The intense peak $(\mathrm{G})$ corresponds to the origin of the ${ }^{\mathbf{1}} \mathrm{A}_{\mathbf{1}}$ state, and Peaks A-F are an extended bending vibrational progression in the ${ }^{3} \mathrm{~B}_{1}$ state. The triplet origin was reported to be peak A, giving a singlet-triplet splitting of $19.5 \mathrm{kcal} / \mathrm{mol}$. Reprinted by permission from the American Chemical Society.

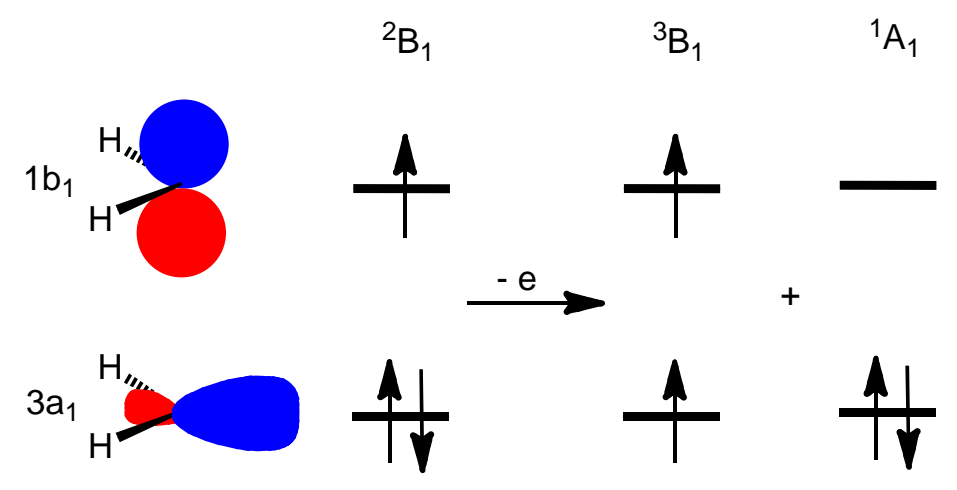

Figure 8. Schematic depiction of formation of the ${ }^{3} \mathrm{~B}_{1}$ and ${ }^{1} \mathrm{~A}_{1}$ states of $\mathrm{CH}_{2}$ by detachment of an electron from the ${ }^{2} \mathrm{~B}_{1}$ state of $\mathrm{CH}_{2}{ }^{\circ-}$.

The observed photoelectron spectrum can be readily understood from a qualitative MO description. As shown schematically in Fig. 8, in both the ${ }^{2} \mathrm{~B}_{1}$ ground state of the radical anion and in the ${ }^{1} \mathrm{~A}_{1}$ state of neutral $\mathrm{CH}_{2}$, two electrons occupy the $3 \mathrm{a}_{1} \mathrm{MO}$, which is largely comprised of a hybridized $\mathrm{AO}$ on carbon. Consequently, very little change in geometry is expected on formation of the lowest singlet state of neutral $\mathrm{CH}_{2}$ by photodetachment of the electron in the $2 \mathrm{p}$ $\mathrm{AO}$ of the radical anion. In contrast, because the ${ }^{3} \mathrm{~B}_{1}$ state has one less electron than the radical anion in the $3 \mathrm{a}_{1} \mathrm{MO}$, a large change in geometry is expected on formation of the triplet state of neutral $\mathrm{CH}_{2}$ by photodetachment of an electron from the $3 \mathrm{a}_{1} \mathrm{MO}$ of the radical anion.

As a consequence of the change in geometry between the ${ }^{2} \mathrm{~B}_{1}$ state of $\mathrm{CH}_{2}{ }^{--}$and the ${ }^{3} \mathrm{~B}_{1}$ state of $\mathrm{CH}_{2}$, an extended progression in the $\mathrm{H}-\mathrm{C}-\mathrm{H}$ bending mode would be expected to be seen in the band corresponding to formation of ${ }^{3} \mathrm{~B}_{1} \mathrm{CH}_{2}$. This is, in fact, is the origin of the $980 \mathrm{~cm}^{-1}$ 
vibrational progression seen in Fig. 7. If the first line in this progression corresponds to a transition from the $v=0$ vibrational level of this mode in $\mathrm{CH}_{2}{ }^{--}$to the $v=0$ vibrational level of this mode in the ${ }^{3} \mathrm{~B}_{1}$ state of $\mathrm{CH}_{2}$, then the energy difference between the ${ }^{1} \mathrm{~A}_{1}$ and ${ }^{3} \mathrm{~B}_{1}$ states of $\mathrm{CH}_{2}$ would, in fact, be $\Delta E_{\mathrm{ST}}=19.5 \pm 0.7 \mathrm{kcal} / \mathrm{mol}$.

However, Harding and Goddard suggested that the first few lines in the triplet region of the NIPE spectrum of $\mathrm{CH}_{2}{ }^{-}$might be hot bands, due to transitions from the $v=1,2$ or 3 excited vibrational levels of $\mathrm{CH}_{2}{ }^{\circ-}$ to the $v=0$ vibrational level of the ${ }^{3} \mathrm{~B}_{1}$ state of $\mathrm{CH}_{2}{ }^{70}$ If the first three lines are, in fact, hot bands, then the fourth line would be the transition from the $v=0$ vibrational level of the $\mathrm{H}-\mathrm{C}-\mathrm{H}$ bending mode in $\mathrm{CH}_{2}{ }^{--}$to the $v=0$ vibrational level of this mode in the ${ }^{3} \mathrm{~B}_{1}$ state of $\mathrm{CH}_{2}$. This reassignment would raise the energy of the $(0,0)$ band for formation of the triplet by ca. $10 \mathrm{kcal} / \mathrm{mol}$, giving $\Delta E_{\mathrm{ST}}=9-10 \mathrm{kcal} / \mathrm{mol}$ in $\mathrm{CH}_{2}$, in good agreement with the best calculated values.

The possibility that some of the lines in the triplet region of the NIPES spectrum were hot bands had not escaped Lineberger and coworkers. They had tested this hypothesis by heating the $\mathrm{CH}_{2}{ }^{-}$ion, before photodetaching it, but the intensities of the bands in the vibrational progression in the ${ }^{3} \mathrm{~B}_{2}$ region of the spectrum remained unchanged. ${ }^{71}$ In order for the Harding and Goddard hypothesis to be consistent with the photodetachment data, the anion vibrational temperature would need to be near $4000 \mathrm{~K}$ and invariant to attempts to modify it. For this reason, it appeared that the hot band explanation was not viable. ${ }^{71}$ Further examination of the anion photoelectron spectrum of $\mathrm{CH}_{2}{ }^{\circ-}$, however awaited the substantial enhancements in electron energy resolution, sensitivity, and chemical and thermal control of the anion source, discussed in Section 3.

When applied to $\mathrm{CH}_{2}{ }^{-}$, the change in the ion source resulted in a dramatic change in the photoelectron spectrum. The new spectrum ${ }^{40}$ (Fig. 9) showed immediately that the initial experiments had been compromised by substantial, unalterable, anion vibrational excitation. The fully analyzed spectrum in Fig. 9 provides a value ${ }^{72}$ of $\Delta E_{\mathrm{ST}}=9.0 \pm 0.1 \mathrm{kcal} / \mathrm{mol}$ for $\mathrm{CH}_{2}$, a result that agrees well with the best values available from calculations. ${ }^{53-56}$ Moreover, all of the extensive vibrational structure in the triplet spectrum could now be definitively assigned as a bending progression in ground state triplet methylene.

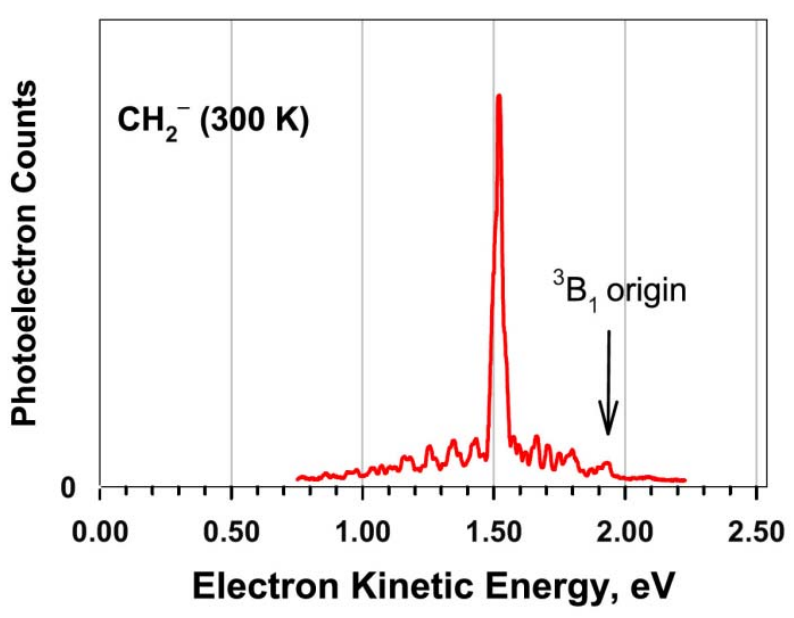

Figure 9. Photoelectron Spectrum of $\mathrm{CH}_{2}{ }^{\circ-}$, obtained with the new anion photoelectron spectrometer. The spectrum directly gives an S-T splitting of $9.0 \pm 0.1 \mathrm{kcal} / \mathrm{mol}^{40}$ The energy 
scale was chosen so as to emphasize the major improvements to the experimental apparatus since the 1976 study.

The substantial change in the H-C-H bend angle on photodetachment to produce triplet methylene results in an extended bending vibrational progression in triplet spectrum. The modest intensity at the triplet origin, the uncontrolled anion excitation and the modest electron energy resolution combined to make the initial assignments incorrect. However, the major instrument improvements described above resulted in a spectrum that gave an unequivocal singlet-triplet splitting without recourse to any fitting of the entire vibrational spectrum. A quantitative fit to the triplet vibration-rotation progression, however, required a more sophisticated analysis, and was accomplished using the rigid bender Hamiltonian developed by Bunker. ${ }^{73}$

When the triplet carbene geometry changes are substantially larger, as in the case of dichlorocarbene, the triplet origin is essentially unobservable and again led to an initially inaccurate assessment of $\Delta \mathrm{E}_{\mathrm{S}-\mathrm{T} .}{ }^{74}$ At this time, a number of methods have been developed to allow the quantitative treatment of extended vibrational progressions with strong mode coupling. ${ }^{75-77}$ These more elaborate analyses could show the inadequacy of the initial dichlorocarbene analysis, and indeed a better S-T splitting was determined, ${ }^{78}$ although with considerably reduced accuracy compared with our normal measurements. The new result was is in much better agreement with high level electronic structure calculations. One important lesson taken from this last study is that large amplitude motions and strongly mixed modes require one to apply a Franck-Condon analysis using vibrational wavefunctions obtained from the multidimensional triplet and anion surfaces. ${ }^{79}$ Such a complete vibrational analysis is not currently feasible with more than four strongly coupled modes.

The studies of $\mathrm{CH}_{2}$ during the 1970s and early 1980s established that calculations could, at least in the case of $\mathrm{CH}_{2}$, make predictions that turned out to be more reliable than the initial interpretations of the experimental data. By the mid-1980s the quality of calculations was such that they now provided an important tool for guiding the interpretation of experimental results. Thus, the calculations and experiments on $\mathrm{CH}_{2}$ changed the culture of physical and physicalorganic chemistry by establishing a synergistic relationship between calculations and experiments.

\section{3. $\Delta E_{\mathrm{ST}}$ in Two Non-Disjoint, Alternant-Hydrocarbon Diradicals and the Effects of} Heteroatom Substitution on the Size of $\boldsymbol{\Delta} \boldsymbol{E}_{\mathrm{ST}}$. As shown in Fig. 10, in meta-benzoquinodimethane (MBQDM), the number of starred carbons exceeds the number of unstarred carbons by two, as is also shown to be the case for TMM in Fig.4. Consequently, like the NBMOs of TMM in Fig. 5, the NBMOs of MBQDM in Fig. 10 are both confined to just the starred set of atoms.

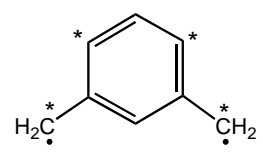

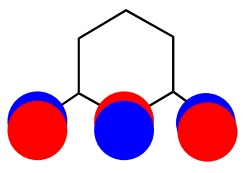

$3 \mathrm{~b}_{1}$

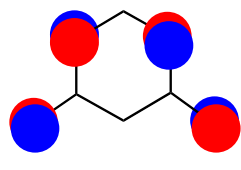

$2 \mathrm{a}_{2}$

Figure 10. Starred and unstarred atoms in and Hückel NBMOs of MBQDM

Since the NBMOs of both TMM and MBQDM are non-disjoint, both diradicals can be predicted to have triplet ground states. ${ }^{1,2}$ Moreover, in order to minimize Coulombic repulsion 
between the open-shell electrons in the singlet states of both diradicals, the singlets can be predicted to utilize different sets of NBMOs than the triplet states.

EPR studies have established that both $\mathrm{TMM}^{80-83}$ and $\mathrm{MBQDM}^{84}$ do, in fact, both have triplet ground states. As described in the following sections, NIPES subsequently provided accurate values for $\Delta E_{\mathrm{ST}}$ in each of these diradicals and also in oxyallyl, a heteroatom derivative of TMM.

4.3.1. $\Delta \boldsymbol{E}_{\mathrm{ST}}$ in TMM. The radical anion of TMM $\left(\mathrm{TMM}^{\circ-}\right)$ was generated in the gasphase by reaction of 1,3-bis(trimethylsilyl)-2-methylenepropane with a mixture of $\mathrm{F}^{-}$and $\mathrm{F}_{2}$ (the Squires reaction). ${ }^{85}$ The NIPE spectrum of the TMM radical anion is shown in Fig. $11 .^{86}$

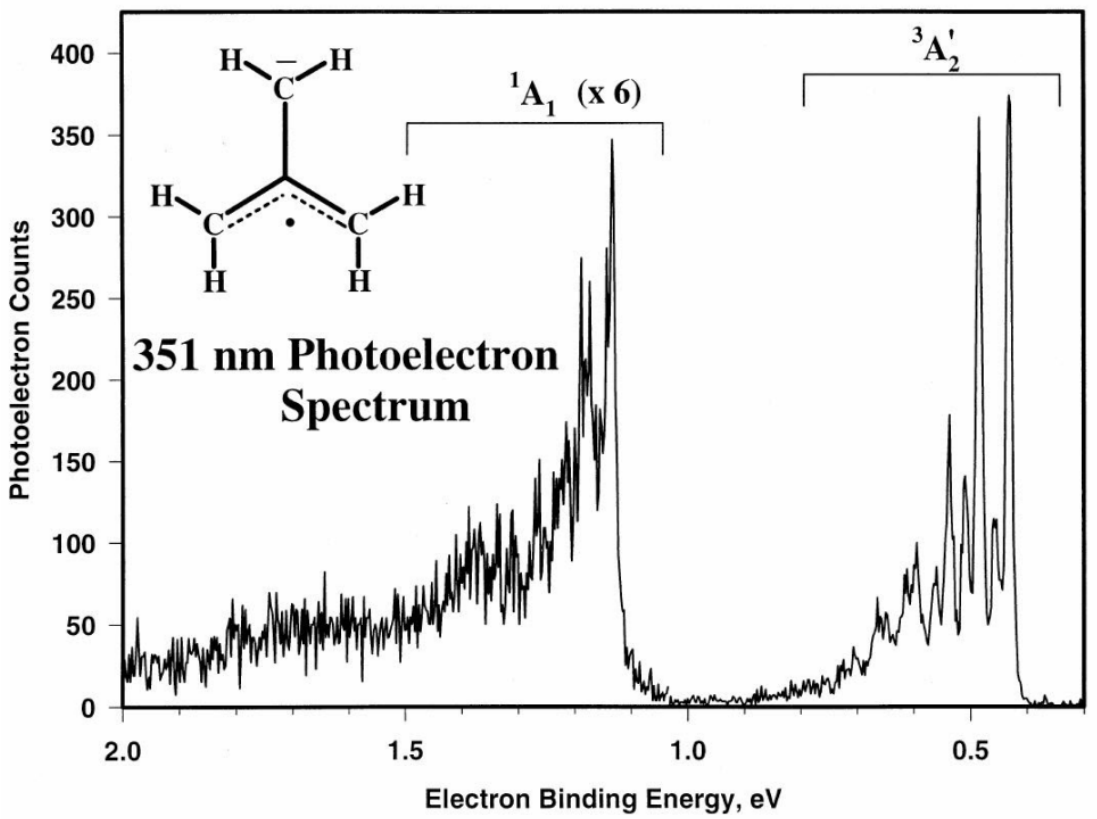

Figure 11. Photoelectron spectrum ${ }^{86}$ of $\mathrm{TMM}^{\bullet-}$ Reprinted from J. Am. Soc. Mass Spectrom., with permission.

As noted in section 2.5, $\mathrm{TMM}^{--}$is planar; but, as shown in Figure 12, the geometry of the lowest singlet state of TMM $\left({ }^{1} \mathrm{~B}_{1}\right)$ has one $\mathrm{CH}_{2}$ group twisted out of conjugation. ${ }^{1,28-33}$ Consequently, photodetachment of an electron from $\mathrm{TMM}^{*-}$ does not lead to the lowest energy geometry of the singlet state of TMM, but, instead, to a planar geometry. 
As shown in Figure 12, there are two planar singlet geometries with $C_{2 v}$ symmetry. One is a ${ }^{1} B_{2}$ state, and the other a ${ }^{1} \mathrm{~A}_{1}$ state. They represent two of the possible Jahn-Teller distortions of the degenerate ${ }^{1} \mathrm{E}^{\prime}$ state of $D_{3 \mathrm{~h}}$ TMM. It has been shown, both by analysis and by calculations,
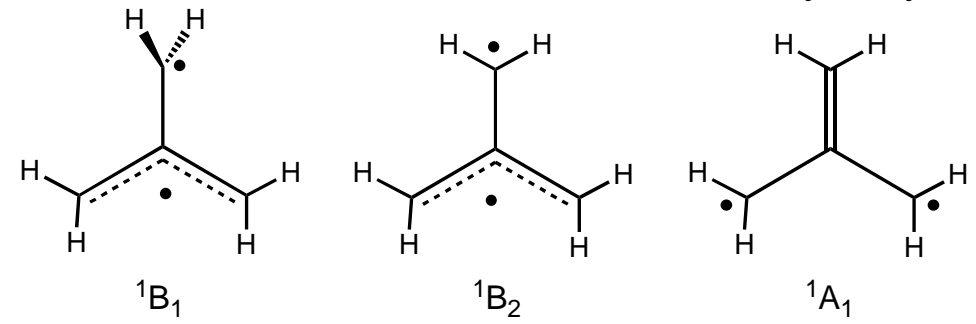

Figure 12. Representations of the bonding in three electronic states of singlet TMM

that the three equivalent minima for pseudorotation of singlet TMM about a $D_{3 \mathrm{~h}}$ geometry are ${ }^{1} \mathrm{~A}_{1}$, and the three transition structures that join the minima are ${ }^{1} \mathrm{~B}_{2} \cdot{ }^{87}$ Therefore, the planar singlet state that is accessed by photodetachment of an electron from $\mathrm{TMM}^{\circ-}$ is, presumably, ${ }^{1} \mathrm{~A}_{1}$.

An important question is whether ${ }^{1} \mathrm{~A}_{1}$ is an energy minimum, a transition structure (TS), or a mountain top on the potential energy surface for singlet TMM. (4/4)CASSCF/6-31G* calculations found that ${ }^{1} \mathrm{~A}_{1}$ is a TS, which, by conrotation of the two equivalent methylene groups, leads to another TS, this one with $C_{2}$ symmetry. ${ }^{88}$ The $C_{2}$ TS connects the ${ }^{1} \mathrm{~B}_{1}$ geometry, shown in Fig. 12, with another ${ }^{1} \mathrm{~B}_{1}$ geometry in which an allylic $\mathrm{CH}_{2}$ group and the nonconjugated $\mathrm{CH}_{2}$ are interchanged.

However, at the (4/4)CASPT2/6-31G* level of theory, where the effects of dynamic electron correlation are included, ${ }^{89}$ the planar ${ }^{1} \mathrm{~A}_{1}$ state is computed to be about $0.1 \mathrm{kcal} / \mathrm{mol}$ lower in energy than the $C_{2}$ singlet geometry. ${ }^{88}$ Therefore, although the region of the singlet potential energy surface around the planar ${ }^{1} \mathrm{~A}_{1}$ state appears to be quite flat, the CASPT2 calculations suggest that ${ }^{1} \mathrm{~A}_{1}$ may actually be a very shallow energy minimum.

This conclusion is consistent with the sharpness of the band in the NIPE spectrum of $\mathrm{TMM}^{\bullet-}$ (Figure 11) that corresponds to formation of the lowest singlet state of planar TMM. If ${ }^{1} \mathrm{~A}_{1}$ were a TS, the short duration of its lifetime would be expected to result in the type of line broadening that actually is seen in the bands for the lowest singlet of oxyallyl (vide infra). ${ }^{6}$

From the NIPE spectrum of $\mathrm{TMM}^{\circ-}$ in Figure 11, an experimental value of $\Delta E_{\mathrm{ST}}=16.1 \pm$ $0.2 \mathrm{kcal} / \mathrm{mol}$ was obtained. The best calculations of the computed energy differences between the ${ }^{3} \mathrm{~A}_{2}{ }^{\prime}$ ground state and the planar ${ }^{1} \mathrm{~A}_{1}$ state, ${ }^{28,29}$ are within $10 \%$ of the experimental value.

\subsubsection{The Effect of Heteroatom Substitution on TMM -- $\Delta E_{S T}$ in Oxyallyl (OXA).} Substitution of an oxygen atom for a $\mathrm{CH}_{2}$ group in TMM gives oxyallyl (OXA). The greater electronegativity of oxygen compared to carbon selectively stabilizes the $2 b_{1}$ NBMO of OXA, relative to the $\mathrm{a}_{2}$ NBMO (Fig. 5). Consequently, in the ${ }^{1} \mathrm{~A}_{1}$ wave function for OXA in eq. $21, \mathrm{c}_{1}{ }^{2}$, the coefficient for the configuration in which the $2 b_{1}$ NBMO is doubly occupied, is larger than $\mathrm{c}_{2}^{2}$, the coefficient for the configuration in which the $\mathrm{a}_{2}$ NBMO is doubly occupied.

$$
\Psi\left({ }^{1} \mathrm{~A}_{1}\right)=\mathrm{c}_{1}^{2}\left(\left|\ldots 2 \mathrm{~b}_{1}^{2}>-\mathrm{c}_{2}^{2}\right| \ldots \mathrm{a}_{2}>\right.
$$

In both the ${ }^{1} \mathrm{~B}_{2}$ and ${ }^{3} \mathrm{~B}_{2}$ states of OXA one electron occupies each NBMO. Therefore, the greater occupancy of the lower energy $2 b_{1} \mathrm{MO}$ in the ${ }^{1} \mathrm{~A}_{1}$ wave function stabilizes this "closedshell" wave function, relative to the "open-shell" singlet and triplet wave functions. Since the ${ }^{1} \mathrm{~A}_{1}$ 
and the ${ }^{1} \mathrm{~B}_{2}$ states have nearly the same energies in TMM, it is easy to predict that ${ }^{1} \mathrm{~A}_{1}$ will be lower in energy than ${ }^{1} \mathrm{~B}_{2}$ in OXA. It is also easy to predict that the energy difference between ${ }^{1} \mathrm{~A}_{1}$ and ${ }^{3} \mathrm{~B}_{2}$ in OXA will be much smaller than that between ${ }^{1} \mathrm{~A}_{1}$ and ${ }^{3} \mathrm{~A}_{2}{ }^{\prime}$ in TMM. ${ }^{90,91}$

Another feature of the bonding in ${ }^{1} \mathrm{~A}_{1}$ that stabilizes it, relative to ${ }^{1} \mathrm{~B}_{2}$ and ${ }^{3} \mathrm{~B}_{2}$, is the greater strength of a $\mathrm{C}=\mathrm{O} \pi$ bond, relative to a $\mathrm{C}=\mathrm{C} \pi$ bond. ${ }^{90,91}$ The ${ }^{1} \mathrm{~A}_{1}$ state of $\mathrm{OXA}$ is computed to have a $\mathrm{C}=\mathrm{O}$ bond length and stretching frequency similar to those in acetone, indicating that the ionic resonance structure in Fig. 13 contributes to the ${ }^{1} \mathrm{~A}_{1}$ wave function about the same amount that this type of structure contributes to the ground state of acetone. ${ }^{92}$<smiles>CCCCCC=O</smiles><smiles>C=C/C=C\[O]</smiles><smiles>C=CC=C[O-]</smiles>

Figure 13 Representations of the bonding in the three electronic states of lowest energy in OXA.

Since the ${ }^{3} \mathrm{~B}_{2}$ state has a larger partial $\pi$ bond to oxygen than the ${ }^{1} \mathrm{~B}_{2}$ state, it can be predicted that both ${ }^{1} \mathrm{~A}_{1}$ and ${ }^{3} \mathrm{~B}_{2}$ should be stabilized, relative to ${ }^{1} \mathrm{~B}_{2}$, on going from TMM to OXA. However, without doing calculations, it cannot be predicted whether ${ }^{1} \mathrm{~A}_{1}$ is lower or higher in energy than ${ }^{3} \mathrm{~B}_{2}$ in OXA.

Although calculations predict that ${ }^{1} \mathrm{~A}_{1}$ is the ground state $\left(\Delta E_{\mathrm{ST}}\right.$ negative) of OXAs that are substituted with alkyl groups, ${ }^{91,93}$ the parent OXA diradical is computed to have $\Delta E_{\mathrm{ST}} \approx 0 .{ }^{90}$ The best calculations performed to-date - (4/4) CASPT2 ${ }^{94}$ and EOM-SF-CCSD(dT), ${ }^{95}$ both with an aug- cc-PVTZ basis set, predict a singlet ground state with $\Delta E_{\mathrm{ST}}=-1.3$ and $-1.5 \mathrm{kcal} / \mathrm{mol}$, respectively.

Experimentally, $\mathrm{OXA}^{\circ}$ - was generated by the reaction of acetone with $\mathrm{O}^{\circ}$. The NIPE spectrum of OXA is shown in Figure $14 .^{6}$ The assignments of the two vibrational progressions in the spectrum were made on the basis calculations of the geometrical parameters that change most in forming the lowest singlet and triplet states of OXA by photodetachment of an electron from $\mathrm{OXA}^{\cdot-}$

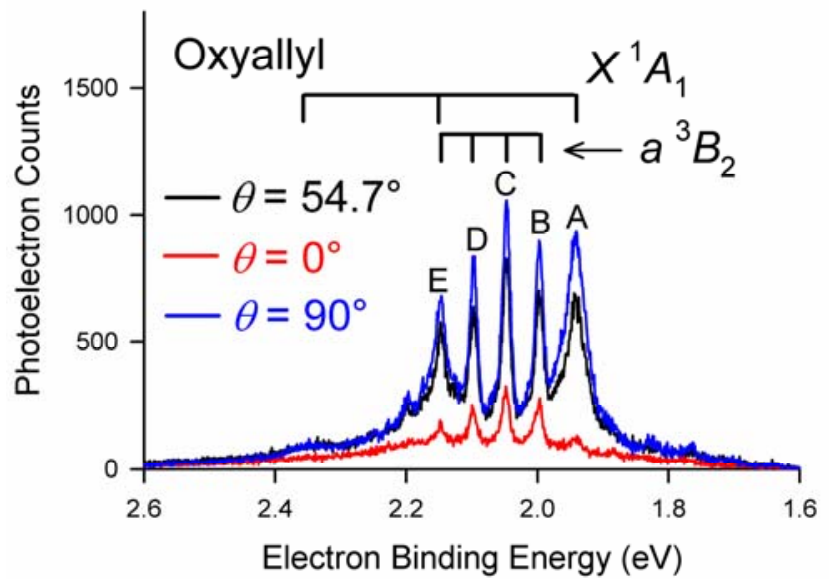

Figure 14. NIPE spectrum ${ }^{6}$ of $\mathrm{OXA}^{\bullet-}$

Reprinted from Angewandte Chemie with permission. 
In forming the ${ }^{3} \mathrm{~B}_{2}$ state the $\mathrm{C}-\mathrm{C}-\mathrm{C}$ bond angle is computed to increase by $7.5^{\circ}$, and a vibrational progression in C-C-C bending, with a splitting of $408 \mathrm{~cm}^{-1}$, is predicted for the triplet. ${ }^{6}$ The actual spacings between the peaks (B, C, D, and part of E) assigned to this progression in the triplet are $405 \pm 10 \mathrm{~cm}^{-1}$. In forming the singlet the $\mathrm{C}=\mathrm{O}$ bond length is calculated to decrease by $0.057 \AA$, and a vibrational progression in $\mathrm{C}=\mathrm{O}$ stretching, with a splitting of $1695 \mathrm{~cm}^{-1}$, is predicted for the singlet. ${ }^{6}$ The actual spacings between the peaks assigned to this progression (A, E, and the broad peak near $2.35 \mathrm{eV}$ ) in the singlet are $1680 \pm 50 \mathrm{~cm}^{-1}$.

The high frequency observed for $\mathrm{C}=\mathrm{O}$ stretching in the singlet confirms the qualitative prediction of a strong $\mathrm{C}=\mathrm{O} \pi$ bond in this state. ${ }^{90,91}$ In addition, the energy difference between the lowest energy peaks in the singlet and triplet vibrational progressions is $1.3 \mathrm{kcal} / \mathrm{mol}$. This spacing, with the singlet peak at lower energy than the triplet, is, perhaps fortuitously, in essentially exact agreement with highest level calculations ${ }^{94,95}$ of $\Delta E_{\mathrm{ST}}$ for OXA.

Another feature of the spectrum in Fig. 14 is that the peaks in the singlet vibrational progression are much broader than those in the vibrational progression that is assigned to the triplet. The large widths of the bands in the singlet were attributed to uncertainty broadening, due to the short lifetime of the singlet; and, in fact, CASPT2 ${ }^{94}$ and EOM-SF-CCSD ${ }^{95}$ calculations find no barrier to disrotatory ring closure ${ }^{1} \mathrm{~A}_{1}$ state of OXA to cyclopropanone. Therefore, ${ }^{1} \mathrm{~A}_{1}$ is not calculated to be an energy minimum but a transition structure. Thus, NIPES has allowed the $\mathrm{C}=\mathrm{O}$ stretching frequency in the transition state for disrotatory ring opening of cyclopropanone to be measured directly, ${ }^{6}$ providing another example of transition state spectroscopy. ${ }^{7,8}$

What prevents this TS from having such a short lifetime that the peaks in the singlet vibrational progression are too broad to be observed in the NIPE spectrum of OXA ${ }^{\bullet}$ ? Ring closure of singlet OXA to cyclopropanone requires the proper phasing of two vibrational modes - disrotation of the $\mathrm{CH}_{2}$ groups and closing of the $\mathrm{C}-\mathrm{C}-\mathrm{C}$ bond angle. In the planar ${ }^{1} \mathrm{~A}_{1}$ state of OXA these two vibrational modes are orthogonal, so that, when the ${ }^{1} \mathrm{~A}_{1}$ state is born the phasing between these two modes is random. It takes time, on the order of $100 \mathrm{fs}$, for $\mathrm{C}-\mathrm{C}-\mathrm{C}$ bending to begin to couple properly to $\mathrm{CH}_{2}$ disrotation, so that ring closure can occur.

This is nicely illustrated in the two-dimensional quantum dynamical simulation of ring closure in ${ }^{1} \mathrm{~A}_{1}$ OXA by Anna Krylov and her coworkers. ${ }^{95}$ During the first $55 \mathrm{fs}$, the $\mathrm{CH}_{2}$ groups disrotate, but this vibration is not coupled to $\mathrm{C}-\mathrm{C}-\mathrm{C}$ bending. During the second $55 \mathrm{fs}, \mathrm{C}-\mathrm{C}-\mathrm{C}$ bending starts to couple to $\mathrm{CH}_{2}$ rotation, and there is a small probability that ring closure will occur after $110 \mathrm{fs}$ have elapsed. In a semi-classical trajectory simulation, closure during the first 110 fs would be due to "direct trajectories", in which the initial coupling of $\mathrm{CH}_{2}$ disrotation and $\mathrm{C}-\mathrm{C}-\mathrm{C}$ bending happens to have the correct phasing for ring closure, wherein the $\mathrm{C}-\mathrm{C}-\mathrm{C}$ bond angle is decreasing as the $\mathrm{CH}_{2}$ groups begin to disrotate out of the molecular plane.

The quantum dynamics simulation by Krylov and coworkers finds that the lifetime of the lowest singlet state of OXA is about $170 \mathrm{fs}$. This calculated lifetime is consistent with the ca. 200 $\mathrm{cm}^{-1}$ line broadening that is observed in those peaks in the NIPE spectrum of OXA ${ }^{\bullet}$ which correspond to formation of the singlet ground state of OXA.

4.3.3 $\Delta E_{\mathrm{ST}}$ in MBQDM. Like the triplet NBMOs of TMM in Fig. 5, the triplet NBMOs of MBQDM in Fig. 10 are non-disjoint. Therefore, like TMM, MBQDM can be predicted have a triplet ground state; and the optimal NBMOs for the two lowest singlet states of MBQDM should 
be different from each other and from the NBMOs for the triplet state. However, unlike the case in $D_{3 \mathrm{~h}} \mathrm{TMM}$, in which the lowest singlet state is a doubly degenerate ${ }^{1} \mathrm{E}$ ' state, the $C_{2 \mathrm{v}}$ symmetry of MBQDM makes it highly unlikely that the "closed-shell" ${ }^{1} \mathrm{~A}_{1}$ wavefunction and the "openshell" ${ }^{1} \mathrm{~B}_{2}$ wave function will have the same energies. Therefore, the first two questions that must be addressed about MBQDM are (a) what are the optimal MOs for these two singlet states and (b) which of the two is lower in energy.

One way to reduce the overlap between the $2 a_{2}$ and $3 b_{1}$ Hückel NBMOs for MBQDM in Fig. 10 is to localize the $2 a_{2}$ NBMO, so that it spans just two carbons of the six-membered ring and does not extend onto the exocyclic $\mathrm{CH}_{2}$ groups. The bonding in the ${ }^{1} \mathrm{~B}_{2}$ state of MBQDM should thus resemble an allyl radical that is attached to the two unstarred carbons of a pentadienyl radical, with little electron delocalization between the two radicals. The bonding in the ${ }^{1} \mathrm{~B}_{2}$ state is depicted schematically in Fig. 15 .

In the ${ }^{1} \mathrm{~A}_{1}$ state of MBQDM, it is not the $2 \mathrm{a}_{2}$ and $3 \mathrm{~b}_{1}$ NBMOs that tend to be confined to different regions of space, but the GVB orbitals derived from them. The GVB MOs are the sum and difference of the $2 a_{2}$ and $3 b_{1}$ NBMOs. Therefore, in order to minimize the spatial overlap between the GVB orbitals, the optimal $2 \mathrm{a}_{2}$ and $3 \mathrm{~b}_{1}$ NBMOs for the ${ }^{1} \mathrm{~A}_{1}$ state should be confined
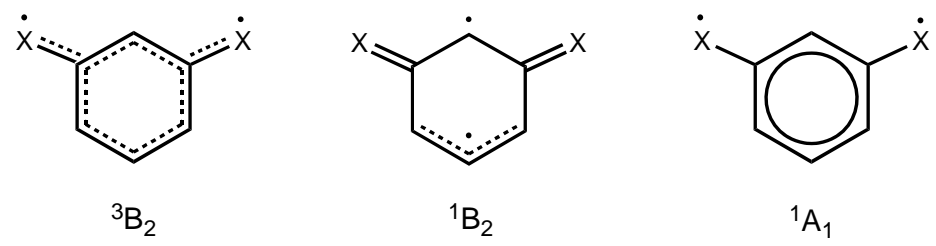

to the same set of atoms.

Figure 15. Schematic depiction of the bonding in the three lowest electronic states of MBQDM $\left(\mathrm{X}=\mathrm{CH}_{2}\right)$ and in MBQ $(\mathrm{X}=\mathrm{O})$. In order to avoid the high Coulombic repulsion that is associated with two electrons of opposite spin appearing in the same region of space, the nonbonding electrons are more localized in the singlet states, ${ }^{1} \mathrm{~B}_{2}$ and ${ }^{1} \mathrm{~A}_{1}$, than in the triplet state, ${ }^{3} \mathrm{~B}_{2}$, of MBQDM and of MBQ.

Inspection of Fig. 10 shows that the $2 a_{2}$ and $3 b_{1}$ Hückel NBMOs have only the $2 p-\pi$ AOs on two exocyclic $\mathrm{CH}_{2}$ groups in common. If the $2 \mathrm{a}_{2}$ and $3 \mathrm{~b}_{1} \mathrm{NBMOs}$ for the ${ }^{1} \mathrm{~A}_{1}$ state of MBQDM are the symmetry combinations of just these two AOs, one GVB orbital will be localized on one of the exocyclic $\mathrm{CH}_{2}$ groups and the second GVB orbital will be localized on the other exocyclic $\mathrm{CH}_{2}$ group. Therefore, the bonding in the ${ }^{1} \mathrm{~A}_{1}$ state of MBQDM can be represented as shown in Fig. 15.

Which of the singlet states, ${ }^{1} \mathrm{~B}_{2}$ or ${ }^{1} \mathrm{~A}_{1}$, is lower in energy in MBQDM? Looking at the depictions of the bonding in these two states in Fig. 15, it can be seen that the $\pi$ bonding in the ${ }^{1} \mathrm{~B}_{2}$ state disrupts the aromaticity of the benzene ring; whereas, the $\pi$ bonding in the ${ }^{1} \mathrm{~A}_{1}$ state does not. Therefore, it is easy to guess that the ${ }^{1} \mathrm{~A}_{1}$ state will be lower in energy than the ${ }^{1} \mathrm{~B}_{2}$ state; and $a b$ initio calculations show this conjecture to be correct. ${ }^{96,97}$ The results of (8/8)CASPT2/6-31G(d) calculations predict that ${ }^{3} \mathrm{~B}_{2}$ is the ground state of MBQDM, with ${ }^{1} \mathrm{~A}_{1}$ $11.0 \mathrm{kcal} / \mathrm{mol}$ higher in energy than ${ }^{3} \mathrm{~B}_{2}$ and ${ }^{1} \mathrm{~B}_{2}$ another $11.9 \mathrm{kcal} / \mathrm{mol}$ higher than ${ }^{1} \mathrm{~A}_{1}{ }^{97}$

Experimentally, the radical anion of MBQDM (MBQDM $\left.{ }^{\circ}\right)$ was produced in the gasphase by the reaction of $m$-xylene with $\mathrm{O}^{*}$, and the NIPE spectrum of MBQDM ${ }^{*}$ is shown in 
Fig. $16 .^{98}$ The spectrum gives values of $\Delta E_{\mathrm{ST}}=9.6 \pm 0.2$ and $<21.5 \mathrm{kcal} / \mathrm{mol}$ for the lowest two singlet states. As was the case with TMM, CASPT2/6-31G(d) calculations on MBQDM give values of $\Delta E_{\text {ST }}$ between ${ }^{3} \mathrm{~B}_{2}$ and both ${ }^{1} \mathrm{~A}_{1}$ and ${ }^{1} \mathrm{~B}_{2}$ that are higher than the values measured, but only by a little more than $1 \mathrm{kcal} / \mathrm{mol}$.

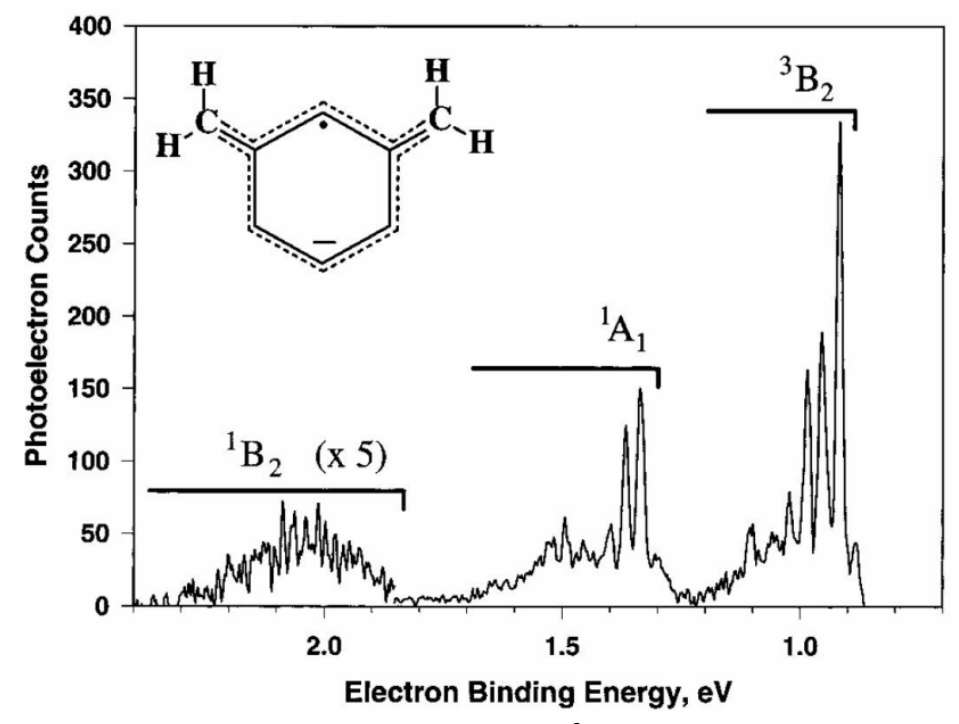

Figure 16 NIPE spectrum ${ }^{98}$ of MBQDM ${ }^{\circ-}$. Reprinted with permission.

4.3.4. $\Delta E_{\mathrm{ST}}$ in meta-Benzoquinone (MBQ). Based on the effect of substituting the oxygen in OXA for a $\mathrm{CH}_{2}$ in TMM, one would expect that substituting the two oxygen atoms in MBQ for the exocyclic $\mathrm{CH}_{2}$ groups in MBQDM would lower $\triangle E_{\mathrm{ST}}$ and might even make a singlet the ground state of MBQ. However, this expectation is not borne out by the results of $a b$ initio calculations on MBQ. CASPT2/6-31G* calculations predict a value of $\Delta E_{\mathrm{ST}}=11.9$ $\mathrm{kcal} / \mathrm{mol}$ between the lowest singlet and triplet state of MBQ, a value that is actually 0.9 $\mathrm{kcal} / \mathrm{mol}$ higher than that in MBQDM. ${ }^{97}$

Although the value of $\Delta E_{\mathrm{ST}}$ changes very little on replacing the two exocyclic methylene groups in MBQDM with the pair of oxygen atoms in MBQ, the relative energies of the two singlet states change by $22.9 \mathrm{kcal} / \mathrm{mol}$. As shown schematically in Fig. 17, in MBQDM ${ }^{1} \mathrm{~B}_{2}$ is calculated to be higher in energy than ${ }^{1} \mathrm{~A}_{1}$ by $11.9 \mathrm{kcal} / \mathrm{mol}$, but in $\mathrm{MBQ}{ }^{1} \mathrm{~B}_{2}$ is computed to be lower in energy than ${ }^{1} \mathrm{~A}_{1}$ by $11.0 \mathrm{kcal} / \mathrm{mol}$. ${ }^{1} \mathrm{~B}_{2}$ is also stabilized relative to the ${ }^{3} \mathrm{~B}_{2}$ state; it goes from being $22.9 \mathrm{kcal} / \mathrm{mol}$ higher than the triplet in MBQDM to $11.9 \mathrm{kcal} / \mathrm{mol}$ higher in MBQ. However, this $11.0 \mathrm{kcal} / \mathrm{mol}$ of selective stabilization of ${ }^{1} \mathrm{~B}_{2}$ in going from MBQDM to MBQ is insufficient to make the energy difference between ${ }^{1} \mathrm{~B}_{2}$ and ${ }^{3} \mathrm{~B}_{2}$ in MBQ smaller than the energy difference between ${ }^{1} \mathrm{~A}_{1}$ and ${ }^{3} \mathrm{~B}_{2}$ in MBQDM. 


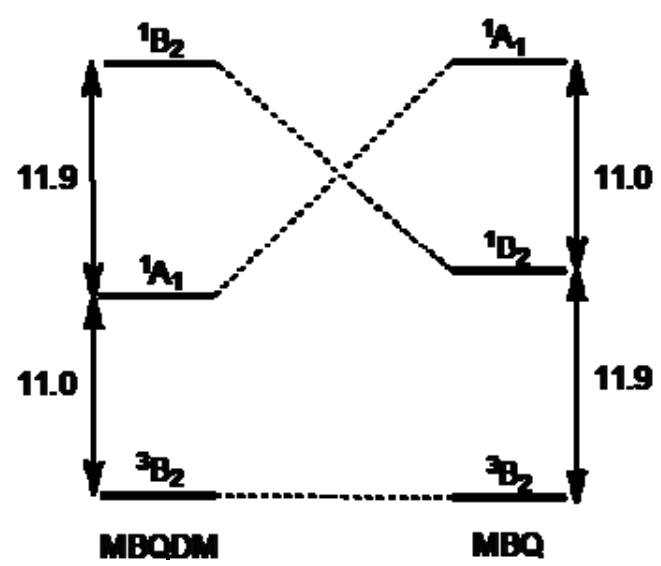

Figure 17. Relative energies of the three lowest electronic states of MBQDM and MBQ.

What causes the large change in the energy of ${ }^{1} \mathrm{~B}_{2}$, relative to both ${ }^{1} \mathrm{~A}_{1}$ and ${ }^{3} \mathrm{~B}_{2}$ ? As noted in the discussion in Section 4.3.2 of the effect of oxygen substitution on making ${ }^{1} \mathrm{~A}_{1}$ the ground state of OXA, part of the stabilization of ${ }^{1} \mathrm{~A}_{1}$, relative to ${ }^{3} \mathrm{~B}_{2}$ on going from TMM to OXA comes from the greater strength of a $C=O \pi$ bond, compared to a $C=C \pi$ bond. The ${ }^{1} A_{1}$ state of TMM has a stronger $\pi$ bond than the ${ }^{3} \mathrm{~B}_{2}$ state to the $\mathrm{CH}_{2}$ group, at which oxygen is substituted in OXA. Therefore, ${ }^{1} \mathrm{~A}_{1}$ is stabilized relative to ${ }^{3} \mathrm{~B}_{2}$ in $\mathrm{OXA}$, and ${ }^{1} \mathrm{~A}_{1}$ and ${ }^{3} \mathrm{~B}_{2}$ are stabilized relative to ${ }^{1} \mathrm{~B}_{2}$ in OXA, because the ${ }^{1} \mathrm{~B}_{2}$ state has no $\pi$ bonding to the $\mathrm{CH}_{2}$ group in TMM at which $\mathrm{O}$ is substituted in OXA.

The same effect operates on going from MBQDM to MBQ. As shown in Fig. 15, in MBQDM the ${ }^{1} \mathrm{~B}_{2}$ state has the strongest $\pi$ bonds to the exocyclic $\mathrm{CH}_{2}$ groups. ${ }^{3} \mathrm{~B}_{2}$ has less $\pi$ bonding to the $\mathrm{CH}_{2}$ groups in MBQDM than ${ }^{1} \mathrm{~B}_{2}$, but more than ${ }^{1} \mathrm{~A}_{1}$. It makes sense, therefore, that substitution of the two oxygen atoms in MBQ for the two exocyclic methylene groups in MBQDM stabilizes both ${ }^{1} \mathrm{~B}_{2}$ and ${ }^{3} \mathrm{~B}_{2}$, relative to ${ }^{1} \mathrm{~A}_{1}$.

These computational predictions have not yet tested. However, three groups have reported the generation of $\mathrm{MBQ}^{\cdot-},{ }^{99-101}$ so it should be just a matter of time until a NIPE spectrum of the radical anion will have been obtained and analyzed. A high-frequency vibrational progression in $\mathrm{C}=\mathrm{O}$ stretching would, presumably, serve as a signature for the ${ }^{1} \mathrm{~B}_{2}$ state in the NIPE spectrum.

4.3.5. Predicted Effects of Heteroatom Substitution at Ring Carbons of MBQDM.

As shown in Figure 10, both of the NBMOs of MBQDM have density on the exocyclic methylene groups; but the NBMOs each have density on different ring carbons. Therefore, substitution of $\mathrm{NH}^{+}$for $\mathrm{CH}$ at one of these ring atoms should provide a large amount of stabilization for just one of the NBMOs. If the selective stabilization of one NBMO is large enough, both non-bonding electrons will tend to occupy that NBMO; and a singlet is likely to be the ground state of the nitrogen-substituted MBQDM derivative.

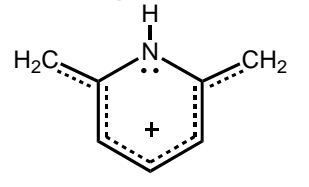

A<smiles></smiles>

B<smiles></smiles>

Figure 18. Three derivatives of MBQDM in which a $\mathrm{CH}$ is replaced by a $\mathrm{NH}^{+}$. 
Both Dougherty and coworkers ${ }^{102}$ and Wang and Krylov ${ }^{103}$ have confirmed this qualitative prediction by carrying out $a b$ initio calculations on the three molecules shown in Fig. 18. Molecule $\mathbf{A}$ is predicted to have a singlet ground state. (Calculations by Cramer and coworkers ${ }^{104}$ have also found that substitution of $\mathrm{NH}_{2}{ }^{+}$for $\mathrm{CH}_{2}$ in $\mathrm{TMM}$ changes the predicted ground state from a triplet to a singlet.) In $\mathbf{B}$, where nitrogen substitution is made at a carbon that belongs to the more delocalized of the two MBQDM NBMOs in Fig. 10, the singlet and triplet are computed to have nearly the same energies. In $\mathbf{C}$ nitrogen substitution is made at a carbon that has no density in either NBMO, and the triplet is computed to remain the ground state.

Dougherty and coworkers pointed out that the effects of the nitrogen substitution in $\mathbf{A}$ - $\mathbf{C}$ can be easily rationalized in VB theory by noting that, as shown in Fig. 18, A and $\mathbf{B}$ can be regarded as weakly perturbed carbocations with a lone pair of electrons localized on $\mathrm{N}$. The MO that is left empty in A and $\mathbf{B}$ is the NBMO in Fig., 10 that is not stabilized by addition of a proton to the nucleus of one of the ring carbon atoms of MBQDM. In contrast, $\mathbf{C}$, like MBQDM, remains a non-Kekulé diradical, a molecule for which no classical resonance structure can be written in which all of the $\pi$ electrons are involved in forming bonds.

\subsection{Two Disjoint, Alternant-Hydrocarbon Diradicals with Singlet Ground States.}

Like TMM and MBQDM, tetramethyleneethane (TME) and 1,2,4,5tetramethylenebenzene (TMB) are both alternant hydrocarbon diradicals for which no classical Kekulé structures can be written. However, in contrast to the non-Kekulé hydrocarbon diradicals TMM and MBQDM, where the number of starred exceeds the number of unstarred atoms by two, Figure 19 shows that in TME and TMB the number of starred atoms is equal to the number of unstarred atoms

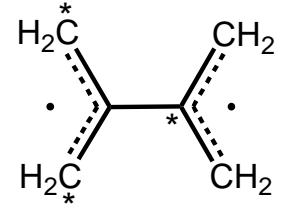

TME

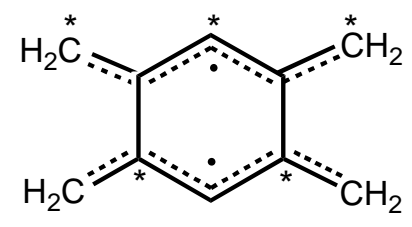

TMB

Figure 19. Starred and unstarred carbons in tetramethyleneethane (TME) and tetramethylenebenzene (TMB).

As discussed in Section 2.5, in alternant hydrocarbon diradicals, such as CBD, in which the number of starred and unstarred atoms is equal, the NBMOs can be chosen to be disjoint, with one NBMO confined to just the starred set of atoms, and the other NBMO confined to just the unstarred set. If the NBMOs, $\psi_{\mathrm{x}}$ and $\psi_{\mathrm{y}}$, of a diradical are disjoint, $K_{\mathrm{xy}} \approx 0$; and the singlet and the triplet states are, to a first approximation, degenerate. ${ }^{1,2}$ Then, dynamic spin polarization, ${ }^{16,17}$ can make the singlet the ground state. Therefore, formal violations of Hund's rule $^{9-12}$ are predicted for diradicals, such as CBD, ${ }^{18-22} \mathrm{TME},{ }^{105-109}$ and $\mathrm{TMB},{ }^{110,111}$ in which $\mathrm{n}_{\mathrm{s}}=$ $\mathrm{n}_{\mathrm{u}}$.

4.4.1. Tetramethyleneethane (TME). As shown in Fig. 20, the symmetry-adapted NBMOs for TME are the in-phase and out-of-phase combinations of the $\pi$ NBMOs for the two allylic radicals, of which TME is comprised. In Fig. 20 the NBMOs have been given their symmetry labels in the $D_{2}$ point group, to which the TME diradical belongs at geometries in 
which the dihedral angle between the planes of the allylic radicals is neither $0^{\circ}\left(D_{2 \mathrm{~h}}\right.$ geometry $)$ nor $90^{\circ}\left(D_{2 \mathrm{~d}}\right.$ geometry).

In TME, because the number of starred and unstarred atoms is the same, it should be possible to choose the NBMOs, so that one NBMO is confined to just the starred set of atoms and the other to just the unstarred set; but the symmetry-adapted, $a_{1}$ and $b_{1}$ NBMOs, shown in Fig. 20, are obviously not disjoint. However, as also shown in Fig. 20, the GVB orbitals, which are the sum and difference of the $\mathrm{a}_{1}$ and $\mathrm{b}_{1}$ NBMOs, are indeed disjoint. ${ }^{1,2}$

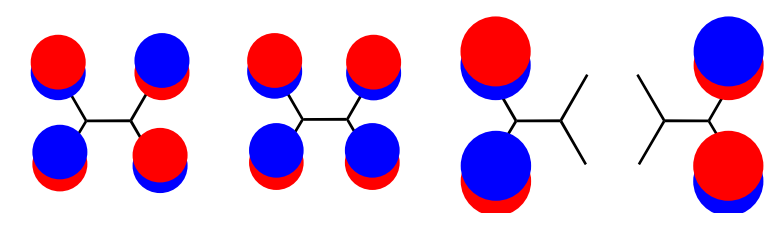

Figure 20. The $a_{1}$ and $b_{1}$ NBMOs of TME and the GVB MOs, which are the sum and the difference of the $a_{1}$ and $b_{1}$ NBMOs.

If one nonbonding electron occupies one GVB orbital of TME and the other nonbonding electron occupies the second GVB orbital, since the GVB orbitals of TME are confined to different regions of space, the lowest singlet and triplet states of TME should have approximately the same energy. However, there are two mechanisms by which the singlet can fall below the triplet in energy. ${ }^{105-109}$

First, the $a_{1}$ and $b_{1}$ orbitals are not degenerate by symmetry, and long-range bonding can result in one NBMO being lower in energy than the latter. For example, if the two allylic radical halves of TME are coplanar or nearly so, long range bonding between the terminal atoms on the allylic radicals makes the $a_{1}$ NBMO is lower in energy than the $b_{1}$ NBMO. In contrast, at dihedral angles, $\phi$, between the two allylic radical halves, near $\phi=90^{\circ}$, spiroconjugation between the $2 \mathrm{p}$ $\pi$ AOs on the terminal atoms reverses the relative energies of the $a_{1}$ and $b_{1}$ NBMOs.

At dihedral angles near $\phi=0^{\circ}$, where the $a_{1}$ NBMO is lower in energy than the $b_{1}$ NBMO, the singlet can take advantage of this difference in orbital energies by making $\mathrm{c}_{1}{ }^{2}$ for the $a_{1}$ NBMO slightly larger than $c_{2}^{2}$ for the $b_{1}$ NBMO in the two-configuration wave function for the lowest singlet state of TME.

$$
\Psi_{T C}=c_{1}^{2}\left|\ldots \psi\left(a_{1}\right)^{2}\right\rangle-c_{2}^{2}\left|\ldots \psi\left(b_{1}\right)^{2}\right\rangle
$$

On the other hand, at dihedral angles near $\phi=90^{\circ}$, where the $b_{1}$ NBMO is lower in energy than the $\mathrm{a}_{1} \mathrm{NBMO}, \mathrm{c}_{2}^{2}>\mathrm{c}_{1}^{2}$ in eq. 21 .

In contrast to the case in the lowest singlet state of TME, in the triplet, one electron occupies each NBMO at all dihedral angles. Therefore, long range bonding interactions, which stabilize the $\mathrm{a}_{1}$ NBMO at dihedral angles close to $\phi=0^{\circ}$ and the $\mathrm{b}_{1}$ NBMO at dihedral angles close to $\phi=90^{\circ}$, can be predicted to make the singlet the ground state of TME.

At some dihedral angle, approximately half way between $\phi=0^{\circ}$ and $\phi=90^{\circ}$, the $\mathrm{a}_{1}$ and $b_{1}$ NMBOs must have the same energy; so at this geometry long-range bonding interactions cannot make the singlet the ground state of TME. In fact, this geometry, where there is no bonding between the NBMOs of the individual allyllic radical halves of TME, is computed to be the equilibrium geometry of the triplet. ${ }^{105-109}$ 
However, there is another interaction between the two allylic radical halves of TME that should selectively stabilize the singlet state, even at the equilibrium geometry of the triplet state. As noted in connection with the discussion of singlet TMM in Section 2.5, there is a negative spin density in the $2 \mathrm{p}-\pi \mathrm{AO}$ at the central carbon of an allylic radical. ${ }^{27}$ These negative spin densities in the two allylic radical halves of TME are anti-parallel in the singlet state, which results in weak $\pi$ bonding between the $2 p-\pi$ AOs on the two central carbons of singlet TME. This bonding interaction is maximal when the allylic radical halves of TME are coplanar at $\phi=0^{\circ}$, but this interaction still exists at dihedral angles between the allylic radicals, other than $\phi$ $=90^{\circ}$. Interaction of the negative spin densities on the central carbons of two allyl radicals, attached to the meta or the para positions of a benzene ring, has been investigated by Lenington and Wenthold. ${ }^{112}$

In contrast, in triplet TME these negative spin densities are anti-parallel, which results in weak antibonding between the $2 \mathrm{p}-\pi$ AOs on the two central carbons of triplet TME, except at dihedral angles that are close to $\phi=90^{\circ}$. Consequently, interaction between the negative spin densities in the $2 p-\pi$ AOs of the central carbons of TME is expected to make the singlet slightly lower in energy than the triplet, even at the equilibrium geometry of the triplet.

Since the equilibrium geometry of the singlet is not likely to be same as the equilibrium geometry of the triplet, the adiabatic value of $\Delta E_{\mathrm{ST}}$ will be even larger in magnitude than the value of $\Delta E_{\mathrm{ST}}$ at the equilibrium geometry of the triplet. Thus, it was claimed that there is no obvious physical mechanism by which the triplet can fall below the singlet state of TME at any geometry. ${ }^{109}$

All the calculations that have been performed found the $D_{2 \mathrm{~d}}\left(\phi=90^{\circ}\right)$ geometry to be the equilibrium geometry of the singlet, and the singlet to be the global TME minimum. ${ }^{105-109}$ On the other hand, some calculations have found that the triplet does fall slightly below the singlet in energy at the equilibrium geometry of the triplet. ${ }^{106,108}$ However, even these latter calculations predict the triplet to be, at best, metastable.

Experimentally, TME was generated in a glassy matrix by Dowd in 1967 and found to have a triplet EPR signal. ${ }^{113} \mathrm{~A}$ subsequent study of the temperature dependence of the intensity of triplet EPR signal found that it followed the Curie-Weiss law. ${ }^{114}$

The linear Curie-Weiss plot does not rule out the possibility that the triplet is only metastable, provided that, at the equilibrium geometry of the triplet, the triplet converts only very slowly to the singlet ground state. Other possible interpretations of the linear Curie-Weiss plot are that the triplet is the ground state or has exactly the same energy as the singlet state. ${ }^{5}$ However the computational results cast doubt on both of these interpretations, and these interpretations appear to be ruled out by the NIPE spectrum of TME* ${ }^{-115}$

The radical anion of TME can be generated by reaction of 2,3-dimethyl-1,3-butadiene with $\mathrm{O}^{\circ-1},{ }^{116}$ and the NIPE spectrum of TME ${ }^{*-}$ is shown in Fig. $21 .{ }^{115}$ Since the three-fold degeneracy of the triplet should make the band for the formation of this state three times as intense as the band for the formation of the singlet state, the NIPE spectrum in Figure 21 indicates that the ground state of TME is a singlet, with $\Delta E_{\mathrm{ST}}=-3 \mathrm{kcal} / \mathrm{mol}$. 


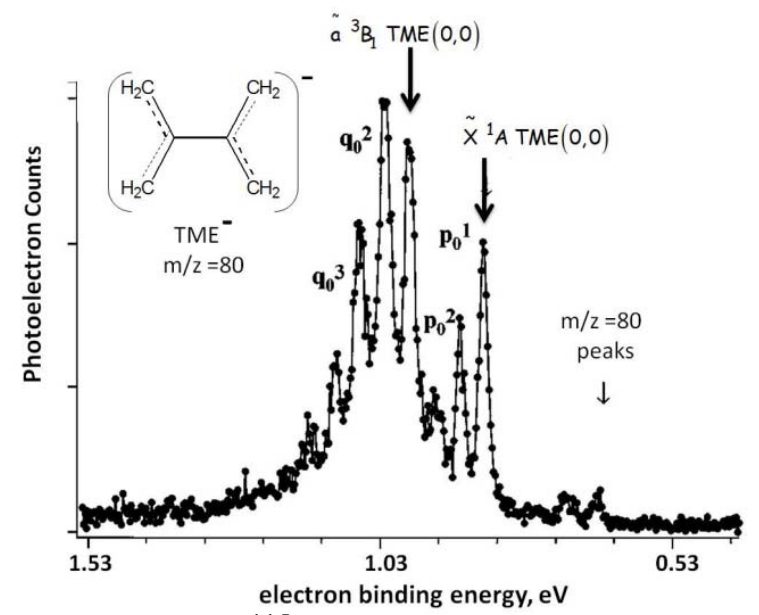

Figure 21 NIPE spectrum ${ }^{115}$ of TME ${ }^{*-}$ Reprinted with permission.

$\mathrm{TME}^{--}$is calculated to have a $D_{2 \mathrm{~d}}$ geometry; and the size of the singlet-triplet energy difference in TME, measured at what is presumably the $D_{2 \mathrm{~d}}$ geometry of TME"- appears to be too large to make it likely that the triplet falls below the singlet at the $D_{2}$ equilibrium geometry of the triplet. ${ }^{105-109}$ Therefore, the only way to reconcile the linear Curie-Weiss plot that was obtained by Dowd with the NIPE spectrum of $\mathrm{TME}^{--}$seems to be to conjecture that the triplet state of TME, when populated, relaxes only very slowly to the lower energy state. ${ }^{108,115}$ Support for this possibility comes from experiments on another diradical. ${ }^{117,118}$ The results of experiments and calculations ${ }^{119}$ on derivatives of TME have been reviewed and discussed. ${ }^{5}$

4.4.2. Tetramethylenebenzene (TMB). Fig, 22 shows the symmetry-adapted NBMOs of TMB. They are the in-phase and out-of-phase combinations of the NBMOs of two pentadienyl radicals. In fact, the lowest singlet and triplet states of TMB are best regarded as two pentadienyl radicals, joined by $\sigma$ bonds between the carbons that lie on nodes of the NBMOs.

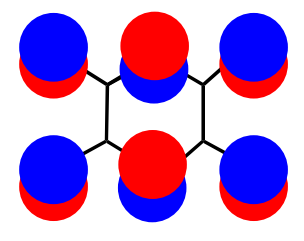

$\mathrm{a}_{2 \mathrm{u}}$

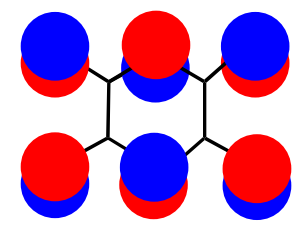

$b_{1 u}$

Figure 22. The $a_{2 u}$ and $b_{1 u}$ NBMOs of TMB. The GVB orbitals of TMB, which are the sum and the difference of the $a_{2} u$ and $b_{1 u}$ NBMOs are disjoint.

Unlike the case in TME, where the one $\mathrm{C}-\mathrm{C} \sigma$ bond that joins the central carbons of the allylic radical halves of TME allows the allylic radicals to rotate with respect to each other, the pair of $\mathrm{C}-\mathrm{C}$ bonds in TMB keeps the pentadienyl radicals coplanar. With the dihedral angle between the pentadienyl radicals fixed at $\phi=0^{\circ}$, TMB lacks the conformational flexibility that complicates the interpretation of the experimental results for TME

As is the case in TME, the symmetry-adapted NBMOs of TMB are not disjoint. However, the GVB orbitals, which are the sum and difference of the $a_{2 u}$ and $b_{1 u}$ NBMOs, are 
disjoint. One GVB orbital is a pentadienyl NBMO that is localized at the top half of the diradical; and the other GVB orbital is a pentadienyl NBMO that is localized at the lower half.

Since the dihedral angle between the planes of the pentadienyl radicals is fixed at $\phi=0^{\circ}$ in TMB, the singlet state of TMB is stabilized, relative to the triplet, by two effects -- throughspace interactions between the pentadienylic NBMOs, which favors the occupancy of the $\mathrm{a}_{2 \mathrm{u}}$ over the $b_{1 u}$ NBMO, and $\pi$ bonding between the negative spin densities at the nodal carbons. The two effects combine to result in a bond distance between the nodal carbons of the two pentadienyl radicals that is computed to be ca. $0.01 \AA$ shorter in the singlet than in the triplet state of TMB and in a calculated value of $\Delta E_{\mathrm{ST}}$ between -5 and $-6 \mathrm{kcal} / \mathrm{mol}$ in this diradical. ${ }^{110}$, 111

The first experiments, designed to test the prediction of a singlet ground state for TMB, were published by Roth and co-workers; and these experiments seemed to show that the prediction was wrong. ${ }^{120,121}$ The experiments found a triplet EPR signal in matrix-isolated TMB; and the intensity of the signal followed the Curie-Weiss law, thus indicating that TMB had a triplet ground state. In addition, the UV-VIS spectrum that was observed better fit the spectrum that had been predicted for the triplet, rather than for the singlet state of TMB. ${ }^{110,111}$

However, subsequent experiments by Berson and coworkers showed that the EPR signal in matrix-isolated TMB did not belong to TMB. ${ }^{122,123}$ The UV-VIS spectrum of TMB could be photo-bleached, but the EPR signal persisted, undiminished in intensity. Berson and coworkers also succeeded in obtaining a ${ }^{13} \mathrm{C}$ NMR spectrum of TMB, and the sharpness of the ${ }^{13} \mathrm{C} N M R$ spectrum was inconsistent with a triplet ground state for TMB. ${ }^{124}$

Roth's experimental results stimulated the recalculation of the UV-VIS spectrum of TMB, this time including the effects of dynamic electron correlation. ${ }^{89}$ (10/10)CASPT2 calculations predicted a UV-VIS spectrum for the singlet that was in good agreement with the observed spectrum. ${ }^{110}$ The new calculations were also able to account for the vibrational structure in the long-wavelength band in the UV-VIS absorption spectrum, which had been observed by both the Roth and Berson groups. ${ }^{120-124}$

The calculations found that, as shown in Fig. 23, although the singlet ground state of TMB can be described as two pentadienyl radicals, joined by a pair of single bonds, the first excited singlet state has an aromatic six-membered ring, with four nonbonding electrons largely localized at the four exocyclic carbons. The change in the $\mathrm{C}-\mathrm{C}$ bond lengths on going from the ground state to the first excited singlet state of TMB is responsible for the vibrational structure seen in the long wavelength UV-VIS absorption of TMB. ${ }^{120-124}$

Although it is now certain that TMB has a singlet ground state, the size of $\Delta E_{\mathrm{ST}}$ in TMB has not yet been measured. It seems likely that, as shown in Figure 24, reaction of 1,2,4,5tetramethylenecyclohexane ${ }^{125}$ or 1,4-dimethylene-2,5-dimethylcyclohexa-2,5-2,5-diene ${ }^{126}$ with $\mathrm{O}^{\circ-}$ would produce the radical anion of TMB. The NIPE spectrum of TMB ${ }^{\circ-}$ should provide an experimental value of $\Delta E_{\mathrm{ST}}$ in TMB, which could be compared with the calculated value of $\Delta E_{\mathrm{ST}}$ $=-5$ to $-6 \mathrm{kcal} / \mathrm{mol}^{110,111}$

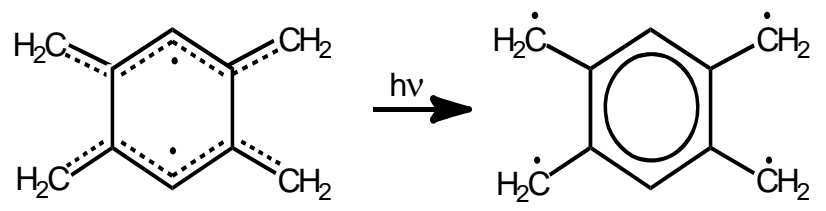

Figure 23. Schematic depiction of the changes in electronic structure of TMB that are predicted to occur on going from the singlet ground state to the first exited singlet state. 


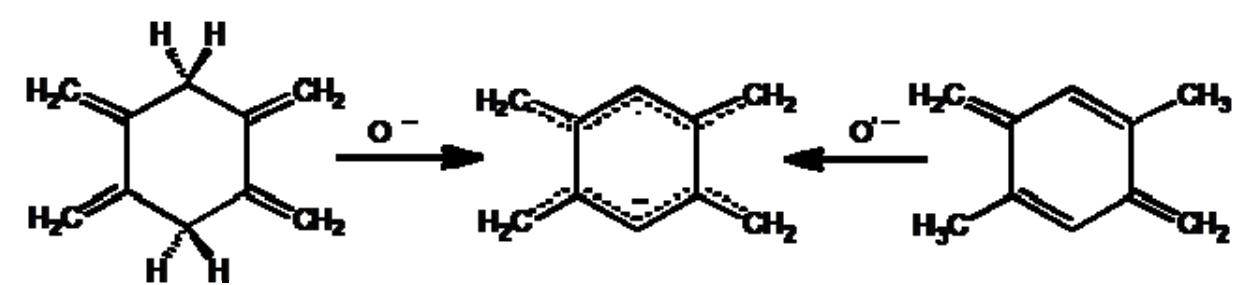

Figure 24. Two possible reactions for generating TMB`- for NIPES.

\subsection{Violation of the Strictest Form of Hund's Rule in $\boldsymbol{D}_{\mathbf{8 h}}$ Cyclooctatetraene (COT).}

As discussed in the two previous sections, TME and TMB are diradicals that have both been found to have singlet ground states. However, as also discussed, in neither of these diradicals does the pair of NBMOs have exactly the same energy. Therefore, it cannot really be claimed that TME and TMB violate the strictest form of Hund's rule.

Hund's rule was originally derived for atoms $;{ }^{9-12}$ and, for a violation of the strictest form of Hund's rule to be claimed, the singly-occupied MOs in a molecular diradical must, like the degenerate pair of singly occupied AOs in an atomic diradical, have exactly the same energy. This means that the singly occupied MOs in a molecular diradical must both belong to the same, two-fold-degenerate representation of the point group for the molecule. Non-Kekulé, hydrocarbon diradicals, such at TME and TMB, do not have high enough symmetry to have NBMOs that are exactly degenerate.

Annulenes with $D_{\text {nh }}$ symmetry do have MOs that come in degenerate pairs; but, in order to have a half-filled shell of MOs, the total number of $\pi$ electrons must be $4 m$, where $m$ is an integer. $(\mathrm{CH})_{4 \mathrm{~m}-1}$ anions, $(\mathrm{CH})_{4 \mathrm{~m}+1}$ cations, and $(\mathrm{CH})_{4 \mathrm{~m} \pm 2}$ dianions and dications do have halffilled shells of degenerate MOs, but the degenerate MOs that are half-filled are not disjoint. ${ }^{51}$ Only $(\mathrm{CH})_{4 \mathrm{~m}}$ annulenes have half-filled shells of disjoint MOs, and the half-filled disjoint MOs only have exactly the same energy at $D_{(4 \mathrm{~m}) \mathrm{h}}$ geometries. ${ }^{1,2,127}$

Unfortunately the $D_{(4 \mathrm{~m}) \mathrm{h}}$ geometries of $(\mathrm{CH})_{4 \mathrm{~m}}$ annulenes are the transition structures for bond shifting in, for example, $\mathrm{CBD}(\mathrm{m}=1)$ and COT $(\mathrm{m}=2)$. Nevertheless, as discussed in section 4.3.2, in connection with OXA, NIPES can be used to access transition state geometries. ${ }^{8}$ Therefore, the Borden and Lineberger groups decided to attempt to obtain the NIPE spectrum of the radical anion of $\mathrm{COT}\left(\mathrm{COT}^{\circ-}\right)$. The detailed reasoning in planning this experiment ${ }^{6}$ was as follows.

Although neutral COT is tub-shaped, $\mathrm{COT}^{--}$is planar. $^{128}$ Therefore, photodetachment of an electron should initially generate planar COT. The triplet state of neutral COT is predicted to have a planar $D_{8 \mathrm{~h}}$ geometry. ${ }^{129,130}$ Since $\mathrm{COT}^{\circ-}$ undergoes a Jahn-Teller distortion to a bondalternated geometry with $D_{4 \mathrm{~h}}$ symmetry, ${ }^{128}$ the difference in geometries between the radical anion and the triplet should lead to the observation in the triplet portion of the NIPE spectrum of a progression in the bond-alternation vibrational mode -- the vibrational mode that shortens four of the C-C bonds in $D_{8 \mathrm{~h}} \mathrm{COT}$ and lengthens four of the others.

In contrast to the planar equilibrium geometry of triplet COT, a planar $D_{4 \mathrm{~h}}$ singlet is the transition structure for COT ring inversion. ${ }^{129,130}$ However, double-well nature of the potential energy surface for ring inversion in the singlet state means that, even at the transition state geometry, the ring inversion vibration should be quantized, although the vibrational levels above the top of the barrier are likely to be closely spaced. Nevertheless, we hoped that in the NIPE 
spectrum of $\mathrm{COT}^{*}$, the peak for formation of the planar $D_{4 \mathrm{~h}}$ singlet transition state for ring inversion of neutral would be sharp enough so that its energy, relative to that of the peak for formation of the $D_{8 h}$ triplet, could be measured.

Of course, the singlet transition structure, whose energy we needed to know in order to confirm the prediction of a violation of the strictest form of Hund's rule, was not the $D_{4 \mathrm{~h}}$ transition structure for ring inversion but the $D_{8 \mathrm{~h}}$ transition structure for bond shifting in planar COT. Fortunately, previous NMR studies had found that bond shifting in COT requires 3 - 4 $\mathrm{kcal} / \mathrm{mol}$ more energy than ring inversion. ${ }^{131-133}$ Therefore, by adding this amount of energy to that measured for formation of the $D_{4 \mathrm{~h}}$ transition structure for ring inversion, we knew that we would be able to obtain $\Delta E_{\mathrm{ST}}$ between the $D_{8 h}$ triplet and the $D_{8 \mathrm{~h}}$ singlet transition state for bond shifting from the NIPE spectrum of COT"*.

The NIPE spectrum of is shown in Fig. 25. ${ }^{7}$ The expected progression in the bond alternation vibration can be seen in the triplet region of the spectrum, along with a much weaker progression in the ring breathing vibration. The same pair of vibrational progressions can also be seen in the singlet region of the spectrum, but the first band in the vibrational progression for bond alternation in the singlet is split into a doublet at $1370 \mathrm{~cm}^{-1}$ and $1670 \mathrm{~cm}^{-1}$.

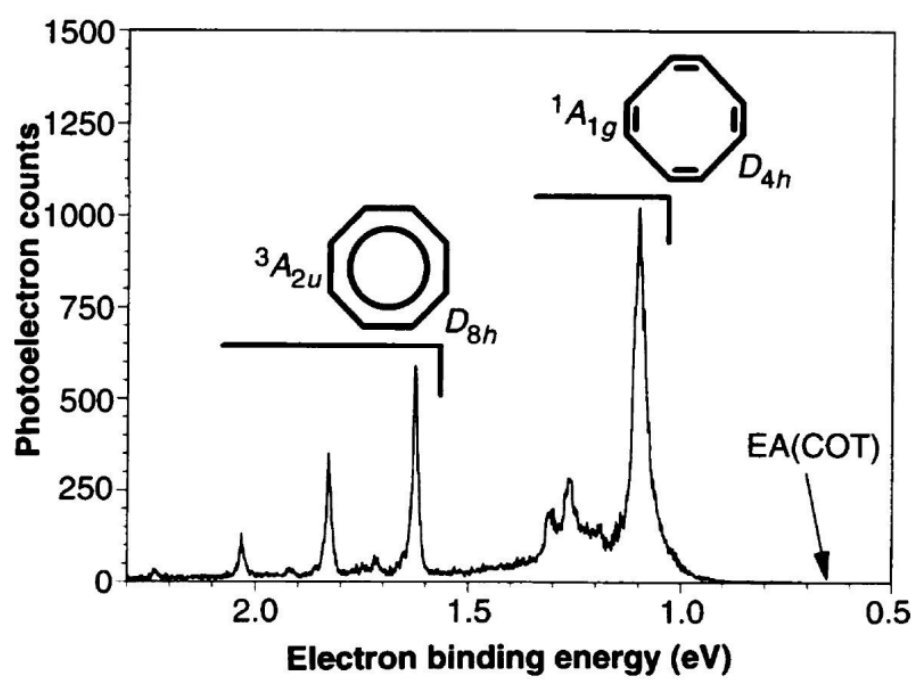

Figure 25. NIPE spectrum of $\mathrm{COT}^{*-7} \cdot$ (Reprinted from Science, with permission) The arrow that is labeled $\mathrm{EA}(\mathrm{COT})$ points to the energy of the adiabatic EA of $\mathrm{COT}^{\circ-}$, which corresponds to formation of $\mathrm{COT}$ at its tub-shaped equilibrium geometry.

Figure 26 provides the explanation for this splitting. The first peak in the vibrational progression for bond alternation in the singlet is calculated to be at $1535 \mathrm{~cm}^{-1}$ above the band for formation of the planar singlet. An energy of $1535 \mathrm{~cm}^{-1}$ corresponds to $4.4 \mathrm{kcal} / \mathrm{mol}$, which puts the calculated energy of the first band in the vibrational progression for bond alternation in the singlet, close to or slightly above the top of the barrier for bond shifting. 


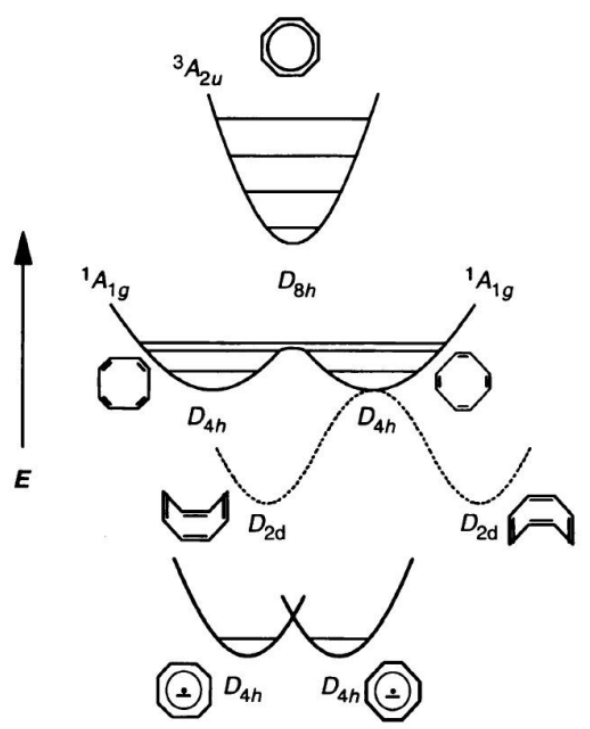

Figure 26. Schematic potential energy surfaces for bond shifting in $\mathrm{COT}^{\circ}$ and in the singlet and triplet states of neutral COT. The dotted line corresponds to the orthogonal vibrational coordinate for ring inversion in the lowest singlet state of $D_{4 \mathrm{~h}}$ COT.

As shown in Fig. 26, at, or close to the top of the barrier for bond shifting, there are actually two vibrational levels that roughly correspond to the in-phases and out-of phase combinations of the first excited vibrational wave function at each of the two minima. The observed $300 \mathrm{~cm}^{-1}$ splitting in the singlet region of the NIPE spectrum is equal to the energy difference between these two vibrational levels, both of which can be accessed from the $D_{4 \mathrm{~h}}$ geometry of the radical anion.

Thus, the unexpected feature in the NIPE spectrum of COT ${ }^{\circ-}$ i.e., the splitting in the vibrational progression for bond alternation in the singlet - confirms the approximate energy of the $D_{8 \mathrm{~h}}$ transition state for bond shifting in singlet COT. Taking the energy of this transition state as being about $4 \mathrm{kcal} / \mathrm{mol}$ above the energy for formation of the bond-alternated, planar, $D_{4 \mathrm{~h}}$ transition structure for ring inversion, the NIPE spectrum in Fig. 24 shows that the $D_{8 \mathrm{~h}}$ transition state for bond shifting in singlet COT is $8-9 \mathrm{kcal} / \mathrm{mol}$ below the energy of the $D_{8 \mathrm{~h}}$ equilibrium geometry of the triplet state. The measured size of violation of Hund's rule in $D_{8 \mathrm{~h}} \mathrm{COT}$ of $8-9$ $\mathrm{kcal} / \mathrm{mol}$ is smaller than that calculated at the CASPT2/6-31G* level of theory, but, again, only by $1-2 \mathrm{kcal} / \mathrm{mol}^{11,12}$

The experimentally confirmed violation of Hund's rule in $D_{8 \mathrm{~h}}$ COT has the same origin as the predicted violation in $D_{4 \mathrm{~h}} \mathrm{CBD}$, discussed in Section 2.4.3. Because the two nonbonding electrons in $D_{8 \mathrm{~h}} \mathrm{COT}$ are confined to disjoint sets of atoms, to a first approximation the lowest singlet and triplet states have the same energy. Dynamic spin polarization stabilizes the nonuniform distribution of electron spin in the NBMOs of the $D_{8 \mathrm{~h}}$ singlet. ${ }^{16,17}$ In contrast, the triplet has a uniform distribution of electron spin at each carbon; so it cannot be stabilized by dynamic spin polarization. Therefore, it is the selective stabilization of $D_{8 \mathrm{~h}}$ singlet COT by dynamic spin polarization that results in $D_{8 \mathrm{~h}}$ COT being a very rare example of a violation of the strictest version of Hund's rule in a molecule. ${ }^{9-12}$

\section{Conclusions}


This Perspective has described the synergism between qualitative theories, calculations, and experiments in establishing the size of $\Delta E_{\mathrm{ST}}$ in a wide variety of organic diradicals, including nitrenes, methylene, non-Kekulé alternant hydrocarbons, and $D_{8 \mathrm{~h}}$ COT. A simple theoretical principle - that electrons of opposite spin tend to minimize their Coulombic repulsion by being localized in different regions of space - has been shown to explain qualitatively a wide variety of phenomenon.

For example, as discussed in Section 4.2.3, this principle explains (a) the much smaller value of $\Delta E_{\mathrm{ST}}$, both predicted ${ }^{45,46,50}$ and measured in $\mathrm{PhN},{ }^{47-49}$ compared to $\mathrm{HN}^{36}$ and $\mathrm{CH}_{3} \mathrm{~N}^{43}$ (b) the bond lengths that are calculated for the open-shell singlet state of $\mathrm{PhN},{ }^{45,46,50}$ and (c) the large differences between the reactivities that are observed for the singlet states of $\mathrm{PhN}$ and PhCH. ${ }^{51,52}$ The ability of the phenyl group to delocalize the $\pi$ nonbonding electron on nitrogen in the open-shell singlet state of $\mathrm{PhN}$, thus confining this $\pi$ electron to a region of space different from that occupied by the $\sigma$ electron of opposite spin, is responsible for all three of these calculated and observed properties of PhN. ${ }^{51,52}$

The minimization of the Coulombic repulsion between electrons of opposite spin, by confining them to different regions of space, is also responsible a number of predictions and observations about non-Kekulé hydrocarbon diradicals These include (a) the difference between the planar geometry of triplet TMM and the preferred geometry of the singlet, which has one $\mathrm{CH}_{2}$ group twisted out of conjugation, ${ }^{1,28,29,87,88}$ (b) the triplet ground states calculated and found for non-Kekulé diradicals with non-disjoint NBMOs (e.g., TMM ${ }^{1,2,28,29,80-83,86,98}$ and $\mathrm{MBQDM}^{84,96-98}$ ) and (c) the singlet ground states calculated and found for non-Kekulé diradicals with disjoint NBMOs (TME ${ }^{105-109,115}$ and $\left.\mathrm{TMB}^{110,111,122-124}\right)$.

It is to be hoped that NIPES on TMB ${ }^{\circ-}$ will be carried out, in order to provide an experimental value of $\Delta E_{\mathrm{ST}}$ in TMB, to compare with the $(10 / 10) \mathrm{CASPT} 2 / 6-31 \mathrm{G}^{*}$ value of $\Delta E_{\mathrm{ST}}$ $=-5.1 \mathrm{kcal} / \mathrm{mol} .{ }^{110}$ In general CASPT $/ 6-31 \mathrm{G}^{*}$ calculations seem capable of providing values of $\Delta E_{\mathrm{ST}}$ that are within $1-2 \mathrm{kcal} / \mathrm{mol}$ of experiment; but another comparison between a calculated and an experimental value of $\Delta E_{\mathrm{ST}}$ would, of course, be desirable.

CASPT2 calculations, albeit with a basis set larger than $6-31 \mathrm{G}^{*}$, are in nearly perfect agreement with experiments regarding the effect on $\Delta E_{\mathrm{ST}}$ of replacing a $\mathrm{CH}_{2}$ group in TMM with the oxygen in OXA. ${ }^{94,95}$ The calculations and the NIPE spectrum of OXA ${ }^{\bullet-}$ both indicate the singlet ground state of OXA is not an energy minimum but the transition structure for ring opening of cyclopropanone. ${ }^{6,95}$ To those organic chemists who have been taught, and who teach their own students, that transition states are too short-lived to be observable, it must come as a shock to learn that the $\mathrm{C}=\mathrm{O}$ stretching frequency in the transition structure for ring opening of cyclopropanone has been accurately measured by NIPES. ${ }^{6}$

The greater strength of a $\mathrm{C}=\mathrm{O} \pi$ bond, compared to a $\mathrm{C}=\mathrm{C} \pi$ bond, is partially responsible for the predicted and observed change in ground state from a triplet in TMM to a singlet in OXA. ${ }^{90,91}$ Paradoxically, the greater strength of a $\mathrm{C}=\mathrm{O} \pi$ bond, compared to a $\mathrm{C}=\mathrm{C} \pi$ bond, is also responsible for the prediction that the size of $\Delta E_{\mathrm{ST}}$ will be little affected by substitution of the oxygen atoms in MBQ for both of the exocyclic methylene groups in MBQDM. ${ }^{97}$ As shown schematically in Fig. 17, although the relative energies of the two lowest singlet states are greatly affected by this substitution, the energy difference between the lower of the two singlets $\left({ }^{1} \mathrm{~A}_{1}\right.$ in MBQDM and ${ }^{1} \mathrm{~B}_{2}$ in MBQ) and the ${ }^{3} \mathrm{~B}_{2}$ ground state is calculated to be largely unaffected. ${ }^{97}$ 
The prediction, ${ }^{97}$ that the value of $\Delta E_{\mathrm{ST}}$ in MBQ will be comparable in size to the value of $\Delta E_{\mathrm{ST}}=9.6 \pm 0.2 \mathrm{kcal} / \mathrm{mol}$ that has already been measured in MBQDM, ${ }^{98}$ will, hopefully, soon be subjected to an experimental test by NIPES, since the generation of $\mathrm{MBQ}^{\bullet-}$ in the gas phase has been reported. ${ }^{99,100}$

Finally, NIPES on $\mathrm{COT}^{--}$has confirmed experimentally ${ }^{7}$ the violation of the strictest version of Hund's rule that had been predicted for the $D_{8 \mathrm{~h}}$ COT diradical. ${ }^{129,130}$ This experiment actually accessed two different transition states on the potential energy surface for the singlet state - the $D_{4 \mathrm{~h}}$ transition state for ring inversion of COT and the $D_{8 \mathrm{~h}}$ transition state for shifting of the double bonds in the planar molecule. The approximate energy of the $D_{8 \mathrm{~h}}$ transition state for double-bond shifting in the singlet state was indicated in the NIPE spectrum of COT ${ }^{\circ-}$ by the splitting of the peak for the first excited level of the vibration for bond alternation. The energy of this $D_{8 \mathrm{~h}}$ transition state, relative to the $D_{4 \mathrm{~h}}$ transition state for ring inversion, was in good agreement with both calculations ${ }^{129,130}$ and with previous NMR experiments on ring inversion and double bond shifting in derivatives of COT.

The prediction and confirmation of the violation of the strictest form of Hund's rule in $D_{8 \mathrm{~h}} \mathrm{COT}^{7}$ provides an excellent example of the synergism between qualitative theory, quantitative calculations, and experiments. Qualitative theory provided the insight that, because the degenerate pair of NBMOs in $D_{8 \mathrm{~h}}$ COT can be chosen to be disjoint, then, at least to a first approximation, $\Delta E_{\mathrm{ST}} \approx 0$ in $D_{8 \mathrm{~h}} \mathrm{COT} .{ }^{2}$ Consideration of the effect of dynamic spin polarization on selectively stabilizing the lowest singlet state ${ }^{16,17}$ then allowed the prediction that the singlet would be the ground state of $D_{8 \mathrm{~h}}$ COT. This qualitative prediction was supported by the results of quantitative calculations. ${ }^{129,130}$

These predictions of a violation of Hund's rule in $D_{8 \mathrm{~h}}$ COT motivated the NIPES experiments on $\mathrm{COT}^{\circ-} .7$ In order to identify the vibrational progressions that were observed in the NIPE spectrum, additional calculations were performed on the lowest singlet and triplet states of COT. The results of these calculations, as well as the results of previous NMR experiments on COT derivatives, ${ }^{131-133}$ led to the identification of the doublet in the NIPE spectrum as being at the correct energy for the $D_{8 \mathrm{~h}}$ transition state for bond shifting in singlet COT.

Qualitative theory then provided the explanation for why the first peak in the progression for the $\mathrm{C}-\mathrm{C}$ bond alternation vibration in singlet COT should be split into a doublet. The observed splitting provided an independent experimental indication of the energy of the $D_{8 \mathrm{~h}}$ transition state for bond shifting in singlet COT. This analysis of the NIPE spectrum ${ }^{7}$ confirmed $^{-}$ the theoretical prediction of the violation of the strictest version of Hund's rule ${ }^{9-12}$ in the $D_{8 \mathrm{~h}}$ COT diradical.

It has been pointed out in previous sections of this perspective that there are several more NIPE spectra that would be of significant interest. One is the NIPE spectrum of $\mathrm{TMB}^{\bullet-}$, in order to test how well CASPT2/6-31G* calculations qualitatively predict the size of the violation of Hund's rule in TMB. ${ }^{110}$ Another NIPE spectrum of considerable interest is that of MBQ•-, in 
order to see whether, as predicted, ${ }^{97}$ the oxygen atoms in MBQ have little effect on changing the value of $\Delta E_{\mathrm{ST}}$ from that in MBQDM. ${ }^{98}$

However, there are other molecules, not discussed in this Perspective, for which the measurement of $\Delta E_{\mathrm{ST}}$ would be of great interest. One such molecule is cyclobutanetetraone $(\mathrm{CO})_{4} \cdot{ }^{134}$ Calculations have predicted that it has a triplet ground state, ${ }^{134-136}$ and a possible precursor of $(\mathrm{CO})_{4}{ }^{--}$for NIPES has been identified. ${ }^{134}$ An experimental test of the rather surprising prediction that this cyclic ketone should have a triplet ground state would be most welcome.

It may well be that future measurements of $\Delta E_{\mathrm{ST}}$ in diradicals will provide additional evidence of the ability of calculations predict accurately the experimental values. If it becomes clear that calculating singlet-triplet energy differences in diradicals is just as accurate and far easier than measuring them, then making such measurements will no longer have quite the importance that it has had over the past 35 - 40 years. However, there will then certainly be new areas of chemistry in which progress depends on the synergy between qualitative theory, quantitative calculations, and direct experiments.

Acknowledgement. WCL is pleased to acknowledge generous support from the National Science Foundation and the Air Force Office of Scientific Research. WTB thanks the National Science Foundation for supporting his research on diradicals for the past 40 years, the Robert A, Welch Foundation for Grant B0027, and his many coworkers and collaborators, particularly Dr. David A. Hrovat and Professors Ernest R. Davidson and Jerome A. Berson. 


\section{REFERENCES}

1. W. T. Borden, in Diradicals, ed. W. T. Borden, Wiley-Interscience, New York, 1982, pp. 172.

2. W. T. Borden and E. R. Davidson, J. Am. Chem. Soc., 1977, 99, 4587-94.

3. K. M. Ervin and W. C. Lineberger, in Advances in Gas Phase Ion Chemistry, eds. N. G. Adams and L. M. Babcock, JAI Press, Greenwich, 1992, vol. 1, pp. 121-66.

4. P. G. Wenthold and W. C. Lineberger, Acc. Chem. Res., 1999, 32, 597-604.

5. W. T. Borden, in Magnetic Properties of Organic Materials, ed. P. M. Lahti, Marcel Dekker, New York, 1999, pp. 61-110.

6. T. Ichino, S. M. Villano, A. J. Gianola, D. J. Goebbert, L. Velarde, A. Sanov, S. J. Blanksby, X. Zhou, D. A. Hrovat, W. T. Borden and W. C. Lineberger, Angew. Chem., Int. Ed. Engl., 2009, 48, 8509-11.

7. P. G. Wenthold, D. Hrovat, W. T. Borden and W. C. Lineberger, Science, 1996, 272, 1456-59.

8. D. M. Neumark, Acc. Chem. Res., 1993, 26, 33-39.

9. W. T. Borden, H. Iwamura and J. A. Berson, Acc. Chem. Res., 1994, 27, 109-16.

10. W. Kutzelnigg, Angew. Chem., Int. Ed. Engl., 1996, 35, 573-86.

11. D. A. Hrovat and W. T. Borden, in Modern Electronic Structure and Applications in Organic Chemistry, ed. E. R. Davidson, World Scientific Publishing, Singapore, 1997, pp. $171-95$.

12. D. A. Hrovat and W. T. Borden, Theochem-J. Mol. Struct., 1997, 398, 211-20.

13. L. Salem and C. Rowland, Angew. Chem., Int. Ed. Engl., 1972, 11, 92-111.

14. V. Bonačić-Koutecký, J. Koutecký and J. Michl, Angew. Chem., Int. Ed. Engl., 1987, 26, $170-89$.

15. W. A. Goddard, T. H. Dunning, W. J. Hunt and P. J. Hay, Acc. Chem. Res., 1973, 6, 36876.

16. W. T. Borden, J. Am. Chem. Soc., 1975, 97, 5968-70.

17. H. Kollmar and V. Staemmler, Theor. Chim. Acta, 1978, 48, 223-39.

18. D. P. Craig, Proc. R. Soc. London, A, 1950, 202, 498-506.

19. R. J. Buenker and Peyerimh.Sd, J. Chem. Phys., 1968, 48, 354. 
20. H. Kollmar and V. Staemmler, J. Am. Chem. Soc., 1977, 99, 3583-87.

21. W. T. Borden, E. R. Davidson and P. Hart, J. Am. Chem. Soc., 1978, 100, 388-92.

22. J. A. Jafri and M. D. Newton, J. Am. Chem. Soc., 1978, 100, 5012-17.

23. A. A. Ovchinnikov, Theor. Chim. Acta, 1978, 47, 297-304.

24. C. A. Coulson and G. S. Rushbrooke, Proc. Camb. Phil. Soc, 1940, 36, 193-200.

25. C. A. Coulson and H. C. Longuethiggins, Proc. R. Soc. London, A, 1947, 192, 16-32.

26. H. C. Longuet-Higgins, J. Chem. Phys., 1950, 18, $265-74$.

27. T. Bally and W. T. Borden, in Reviews in Computational Chemistry, Vol. 13, ed. K. B. Lipowitz, Wiley, New York, 1999, pp. 1-99.

28. C. J. Cramer and B. A. Smith, J. Phys. Chem., 1996, 100, 9664-70.

29. L. V. Slipchenko and A. I. Krylov, J. Chem. Phys., 2005, 123, 084107 [13 pages].

30. J. C. Gilbert and J. R. Butler, J. Am. Chem. Soc., 1970, 92, 7493.

31. M. Jones, M. E. Hendrick, J. C. Gilbert and J. R. Butler, Tetrahedron Letters, 1970, 11, $845-48$.

32. W. von E. Doering and Birladea.L, Tetrahedron, 1973, 29, 499-512.

33. W. von E. Doering and H. D. Roth, Tetrahedron, 1970, 26, 2825.

34. R. J. Celotta, R. A. Bennett, J. Levine, J. L. Hall and M. W. Siegel, Phys. Rev. A, 1972, 6, 631.

35. M. W. Siegel, R. A. Bennett, R. J. Celotta, J. L. Hall and J. Levine, Phys. Rev. A, 1972, 6, 607.

36. P. C. Engelking and W. C. Lineberger, J. Chem. Phys., 1976, 65, 4223-24.

37. A. Kasdan, E. Herbst and W. C. Lineberger, Chem. Phys. Lett., 1975, 31, 78-82.

38. A. Kasdan, E. Herbst and W. C. Lineberger, J. Chem. Phys., 1975, 62, 541-48.

39. P. F. Zittel, G. B. Ellison, S. V. ONeil, E. Herbst, W. C. Lineberger and W. P. Reinhardt, J. Am. Chem. Soc., 1976, 98, 3731-32.

40. D. G. Leopold, K. K. Murray and W. C. Lineberger, J. Chem. Phys., 1984, 81, 1048-50.

41. P. G. Wenthold, in Reviews of Reactive Intermediate Chemistry, eds. M. S. Platz, R. A. Moss and M. Jones, Wiley, New York, 2007, pp. 207-46. 
42. C. Zetzsch and F. Stuhl, Chem. Phys. Lett., 1975, 33, 375-77.

43. M. J. Travers, D. C. Cowles, E. P. Clifford, G. B. Ellison and P. C. Engelking, J. Chem. Phys., 1999, 111, 5349-60.

44. C. R. Kemnitz, G. B. Ellison, W. L. Karney and W. T. Borden, J. Am. Chem. Soc., 2000, 122, 1098-101.

45. S. J. Kim, T. P. Hamilton and H. F. Schaefer, J. Am. Chem. Soc., 1992, 114, 5349-55.

46. D. A. Hrovat, E. E. Waali and W. T. Borden, J. Am. Chem. Soc., 1992, 114, 8698-99.

47. M. J. Travers, D. C. Cowles, E. P. Clifford and G. B. Ellison, J. Am. Chem. Soc., 1992, 114, 8699-701.

48. R. N. McDonald and S. J. Davidson, J. Am. Chem. Soc., 1993, 115, 10857-62.

49. N. R. Wijeratne, M. D. Fonte, A. Ronemus, P. J. Wyss, D. Tahmassebi and P. G. Wenthold, The Journal of Physical Chemistry A, 2009, 113, 9467-73.

50. M. Winkler, J. Phys. Chem. A, 2008, 112, 8649-53.

51. W. T. Borden, N. P. Gritsan, C. M. Hadad, W. L. Karney, C. R. Kemnitz and M. S. Platz, Acc. Chem. Res., 2000, 33, 765-71.

52. W. L. Karney and W. T. Borden, in Advances in Carbene Chemistry, Vol 3, ed. U. H. Brinker, Elsevier, Amsterdam, 2001, pp. 206-51.

53. I. Shavitt, Tetrahedron, 1985, 41, 1531-42.

54. H. F. Schaefer, Science, 1986, 231, 1100-07.

55. W. A. Goddard, Science, 1985, 227, 917-23.

56. J. F. Harrison, in Advances in the Theory of Atomic and Molecular Systems: Conceptual and Computational Advances in Quantum Chemistry, eds. P. Piecuch, J. Maruani, G. Delgado-Barrio and S. Wilson, Springer, New York, 2009, pp. 1-12.

57. G. Herzberg, Proc. R. Soc. London, A, 1961, 262, 291-317.

58. J. F. Harrison and L. C. Allen, J. Am. Chem. Soc., 1969, 91, 807.

59. J. M. Foster and S. F. Boys, Rev. Mod. Phys., 1960, 32, 305-07.

60. C. F. Bender and H. F. Schaefer, J. Am. Chem. Soc., 1970, 92, 4985.

61. E. Wasserman, W. A. Yager and V. J. Kuck, Chem. Phys. Lett., 1970, 7, 409-13. 
62. E. R. Davidson, in Diradicals, ed. W. T. Borden, Wiley-Interscience, New York, 1982, pp. 73-105.

63. H. M. Frey and G. J. Kennedy, J. Chem. Soc., Chem. Comm., 1975, 233-34.

64. H. M. Frey and G. J. Kennedy, J. Chem. Soc. Faraday Trans. 1, 1977, 73, 164-70.

65. J. F. Harrison, Acc. Chem. Res., 1974, 7, 378-84.

66. W. L. Hase and P. M. Kelley, J. Chem. Phys., 1977, 66, 5093-99.

67. S. V. ONeil, H. F. Schaefer and C. F. Bender, J. Chem. Phys., 1971, 55, 162.

68. V. Staemmler, Theor. Chim. Acta, 1973, 31, 49-61.

69. V. Staemmler, Theor. Chim. Acta, 1974, 35, 309-27.

70. L. B. Harding and W. A. Goddard, J. Chem. Phys., 1977, 67, 1777-79.

71. P. C. Engelking, R. R. Corderman, J. J. Wendoloski, G. B. Ellison, S. V. ONeil and W. C. Lineberger, J. Chem. Phys., 1981, 74, 5460-73.

72. D. G. Leopold, K. K. Murray, A. E. S. Miller and W. C. Lineberger, J. Chem. Phys., 1985, 83, 4849-65.

73. P. R. Bunker and T. J. Sears, J. Chem. Phys., 1985, 83, 4866-76.

74. R. L. Schwartz, G. E. Davico, T. M. Ramond and W. C. Lineberger, J. Phys. Chem. A, 1999, 103, 8213-21.

75. R. Borrelli, Chem. Phys. Lett., 2007, 445, 84-88.

76. R. Borrelli and A. Peluso, J. Chem. Phys., 2008, 128, 044303 [7 pages].

77. V. Vanovschi, A. I. Krylov and P. G. Wenthold, Theor. Chem. Acc., 2008, 120, 45-58.

78. S. W. Wren, K. M. Vogelhuber, K. M. Ervin and W. C. Lineberger, Phys. Chem. Chem. Phys., 2009, 11, 4745-53.

79. K. M. Vogelhuber, S. W. Wren, A. B. McCoy, K. M. Ervin and W. C. Lineberger, J. Chem. Phys., 2011, in press.

80. P. Dowd and K. Sachdev, J. Am. Chem. Soc., 1967, 89, 715.

81. P. Dowd, A. Gold and K. Sachdev, J. Am. Chem. Soc., 1968, 90, 2715.

82. P. Dowd, J. Am. Chem. Soc., 1966, 88, 2587. 
83. R. J. Baseman, D. W. Pratt, M. Chow and P. Dowd, J. Am. Chem. Soc., 1976, 98, 572627.

84. B. B. Wright and M. S. Platz, J. Am. Chem. Soc., 1983, 105, 628-30.

85. P. G. Wenthold, J. Hu and R. R. Squires, J. Am. Chem. Soc., 1994, 116, 6961-62.

86. P. G. Wenthold, J. Hu, R. R. Squires and W. C. Lineberger, J. Am. Soc. Mass Spectrom., 1999, 10, 800-09.

87. E. R. Davidson and W. T. Borden, J. Am. Chem. Soc., 1977, 99, 2053-60.

88. S. B. Lewis, D. A. Hrovat, S. J. Getty and W. T. Borden, J. Chem. Soc.-Perkin Trans. 2, 1999, 2339-47.

89. W. T. Borden and E. R. Davidson, Acc. Chem. Res., 1996, 29, 67-75.

90. Y. Osamura, W. T. Borden and K. Morokuma, J. Am. Chem. Soc., 1984, 106, 5112-15.

91. M. B. Coolidge, K. Yamashita, K. Morokuma and W. T. Borden, J. Am. Chem. Soc., 1990, 112, $1751-54$.

92. D. C. Lim, D. A. Hrovat, W. T. Borden and W. L. Jorgensen, J. Am. Chem. Soc., 1994, 116, 3494-99.

93. A. S. Ichimura, P. M. Lahti and A. R. Matlin, J. Am. Chem. Soc., 1990, 112, 2868-75.

94. T. Ichino, S. M. Villano, A. J. Gianola, D. J. Goebbert, L. Velardi, A. Sanov, X. Zhou, D. A. Hrovat, W. T. Borden and W. C. Lineberger, J. Phys. Chem. A, 2011, 115, 1634-49.

95. V. Mozhayskiy, D. J. Goebbert, L. Velarde, A. Sanov and A. I. Krylov, J. Phys. Chem. A, 2010, 114, 6935-43.

96. S. Kato, K. Morokuma, D. Feller, E. R. Davidson and W. T. Borden, J. Am. Chem. Soc., 1983, 105, 1791-95.

97. D. A. Hrovat, M. A. Murcko, P. M. Lahti and W. T. Borden, J. Chem. Soc.-Perkin Trans. 2, 1998, 1037-44.

98. P. G. Wenthold, J. B. Kim and W. C. Lineberger, J. Am. Chem. Soc., 1997, 119, 1354-59.

99. N. R. Wijeratne and P. G. Wenthold, J. Am. Soc. Mass Spectrom., 2007, 18, 2014-16.

100. A. Fattahi, S. R. Kass, J. F. Liebman, M. A. R. Matos, M. S. Miranda and V. M. F. Morais, J. Am. Chem. Soc., 2005, 127, 6116-22.

101. J. Roithová, D. Schröder and H. Schwarz, Angew. Chem., Int. Ed. Engl., 2005, 44, 309296. 
102. A. P. West, S. K. Silverman and D. A. Dougherty, J. Am. Chem. Soc., 1996, 118, 145263.

103. T. Wang and A. I. Krylov, J. Chem. Phys., 2005, 123, 104304 [6 pages].

104. J. B. Li, S. E. Worthington and C. J. Cramer, J. Chem. Soc.-Perkin Trans. 2, 1998, $1045-$ 51.

105. E. Rodriguez, M. Reguero and R. Caballol, J. Phys. Chem. A, 2000, 104, 6253-58.

106. P. Nachtigall and K. D. Jordan, J. Am. Chem. Soc., 1993, 115, 270-71.

107. P. Nachtigall and K. D. Jordan, J. Am. Chem. Soc., 1992, 114, 4743-47.

108. M. Filatov and S. Shaik, J. Phys. Chem. A, 1999, 103, 8885-89.

109. P. Du and W. T. Borden, J. Am. Chem. Soc., 1987, 109, 930-31.

110. D. A. Hrovat and W. T. Borden, J. Am. Chem. Soc., 1994, 116, 6327-31.

111. P. Du, D. A. Hrovat, W. T. Borden, P. M. Lahti, A. R. Rossi and J. A. Berson, J. Am. Chem. Soc., 1986, 108, 5072-74.

112. M. J. Lenington and P. G. Wenthold, J. Phys. Chem. A, 2007, 114, 1334-37.

113. P. Dowd, J. Am. Chem. Soc., 1970, 92, 1066-68.

114. P. Dowd, W. Chang and Y. H. Paik, J. Am. Chem. Soc., 1986, 108, 7416-17.

115. E. P. Clifford, P. G. Wenthold, W. C. Lineberger, G. B. Ellison, C. X. Wang, J. J. Grabowski, F. Vila and K. D. Jordan, J. Chem. Soc. Perkin Trans. 2, 1998, 1015-22.

116. J. H. Lee, P. K. Chou, P. Dowd and J. J. Grabowski, J. Am. Chem. Soc., 1993, 115, 790203.

117. L. C. Bush, L. Maksimovic, X. W. Feng, H. S. M. Lu and J. A. Berson, J. Am. Chem. Soc., 1997, 119, 1416-27.

118. J. A. Berson, Acc. Chem. Res., 1997, 30, 238-44.

119. J. J. Nash, P. Dowd and K. D. Jordan, J. Am. Chem. Soc., 1992, 114, 10071-72.

120. W. R. Roth, R. Langer, M. Bartmann, B. Stevermann, G. Maier, H. P. Reisenauer, R. Sustmann and W. Muller, Angew. Chem., Int. Ed. Engl., 1987, 26, 256-58.

121. W. R. Roth, R. Langer, T. Ebbrecht, A. Beitat and H. W. Lennartz, Chem. Ber.-Recl., 1991, 124, 2751-60. 
122. J. H. Reynolds, J. A. Berson, K. K. Kumashiro, J. C. Duchamp, K. W. Zilm, A. Rubello and P. Vogel, J. Am. Chem. Soc., 1992, 114, 763-64.

123. J. H. Reynolds, J. A. Berson, J. C. Scaiano and A. B. Berinstain, J. Am. Chem. Soc., $1992,114,5866-67$.

124. J. H. Reynolds, J. A. Berson, K. K. Kumashiro, J. C. Duchamp, K. W. Zilm, J. C.

Scaiano, A. B. Berinstain, A. Rubello and P. Vogel, J. Am. Chem. Soc., 1993, 115, 8073-90.

125. D. T. Longone and F. P. Boettcher, J. Am. Chem. Soc., 1963, 85, 3436.

126. T. Koenig and S. Southworth, J. Am. Chem. Soc., 1977, 99, 2807-09.

127. W. T. Borden, J. Chem. Soc D, Chem Comm., 1969, 15, 881-82.

128. J. H. Hammons, D. A. Hrovat and W. T. Borden, J. Am. Chem. Soc., 1991, 113, 4500-05.

129. M. J. S. Dewar and K. M. Merz, J. Phys. Chem., 1985, 89, 4739-44.

130. D. A. Hrovat and W. T. Borden, J. Am. Chem. Soc., 1992, 114, 5879-81.

131. F. A. L. Anet, J. Am. Chem. Soc., 1962, 84, 671.

132. F. A. L. Anet, A. J. R. Bourn and Y. S. Lin, J. Am. Chem. Soc., 1964, 86, 3576-77.

133. J. F. M. Oth, Pure Appl. Chem., 1971, 25, 573-622.

134. X. Zhou, D. A. Hrovat and W. T. Borden, J. Phys. Chem. A, 2010, 114, 1304-08.

135. H. J. Jiao, G. Frapper, J. F. Halet and J. Y. Saillard, J. Phys. Chem. A, 2001, 105, 594547.

136. X. Zhou, D. A. Hrovat, R. Gleiter and W. T. Borden, Mol. Phys., 2009, 107, 863-70. 